\title{
WestVirginiaUniversity
}

THE RESEARCH REPOSITORY @ WVU

Graduate Theses, Dissertations, and Problem Reports

2016

\section{Moment-type Nonparametric Estimation in Some Direct and Indirect Models}

\author{
Fairouz M.Ali Elmagbri
}

Follow this and additional works at: https://researchrepository.wvu.edu/etd

\section{Recommended Citation}

Elmagbri, Fairouz M.Ali, "Moment-type Nonparametric Estimation in Some Direct and Indirect Models" (2016). Graduate Theses, Dissertations, and Problem Reports. 5539.

https://researchrepository.wvu.edu/etd/5539

This Dissertation is protected by copyright and/or related rights. It has been brought to you by the The Research Repository @ WVU with permission from the rights-holder(s). You are free to use this Dissertation in any way that is permitted by the copyright and related rights legislation that applies to your use. For other uses you must obtain permission from the rights-holder(s) directly, unless additional rights are indicated by a Creative Commons license in the record and/ or on the work itself. This Dissertation has been accepted for inclusion in WVU Graduate Theses, Dissertations, and Problem Reports collection by an authorized administrator of The Research Repository @ WVU.

For more information, please contact researchrepository@mail.wvu.edu. 


\title{
Moment-type Nonparametric Estimation in Some Direct and Indirect Models
}

\author{
Fairouz M.Ali Elmagbri \\ Thesis submitted to the \\ Eberly College of Arts and Sciences \\ at West Virginia University \\ in partial fulfillment of the requirements \\ for the degree of \\ Doctor of Philosophy \\ in \\ Mathematics \\ Committee Members \\ Robert Mnatsakanov, Ph.D, Chair \\ Casian Pantea, Ph.D, Co-Chair \\ E.James Harner, Ph.D \\ Adrian Tudorascu, Ph.D \\ Department of Mathematics \\ Morgantown, WV \\ 2016
}

Keywords: Laplace transform inversion; Current status model; Right-censored model

Copyright 2016 Fairouz Elmagbri 


\title{
ABSTRACT \\ Moment-type Nonparametric Estimation in Some Direct and Indirect Models
}

\author{
Fairouz M.Ali Elmagbri
}

In this research, several approximantions of the probability density function, cumulative distribution function in some direct and indirect models are proposed. They are based on the knowledge of the moments and the scaled Laplace transform of the target functions. The upper bounds for the uniform rate of approximations as well as the mean squared errors are established. Two cases when the support of underlying function is bounded and unbounded from above are studied.

Proposed constructions provide new nonparametric estimates of the distribution and the density functions in right censored, current status, mean residual life time and length biased models. Simulation study justifies the consistency of the proposed estimates. 


\section{Acknowledgements}

First of all, I praise God almighty for granting me this opportunity and the capability to proceed successfully in my study.

I also would like to express my sincere gratitude to my advisors Dr. Robert Mnatsakanov and Dr.Gary Ganser for their support throughout my $\mathrm{Ph} . \mathrm{D}$. research. I am extremely fortunate for having them as advisors, their knowledge, guidance, and motivation helped me at all the times to complete my dissertation.

Besides, I would like to thank my PhD thesis committee: Dr. E. James Harner, Dr. Adrian Tudorascu, and Dr.Casian Pantea for their insightful comments, guidance, and encouragement, which helped me to successfully finish up my research.

I would also like to extend my sincere thanks to Dr. Diamond Harvey, and Dr. Michael Mays, who gave me help and support. I take this opportunity to express sincere gratitude to the Department of Mathematic and Department of Statistics faculty members and staff for their help and support.

Lastly, I would like to thank my family: my husband, my parent, brothers and sisters for their endless support throughout this period. 


\section{Contents}

1 Introduction 1

1.1 Complete models . . . . . . . . . . . . . . 2

1.2 Incomplete models . . . . . . . . . . . . . 4

1.3 Moment recovery of density estimation . . . . . . 8

1.4 Organization of dissertation . . . . . . . . . . 10

2 Complete Model 12

2.1 Direct model . . . . . . . . . . . . . . 12

2.1.1 Mean squared error ............... 13

2.1.2 Simulation .............. 21

2.2 Mean residual life time . . . . . . . . . . . 24

3 Incomplete Models 3

3.1 Right censored model . . . . . . . . . . . . . . 31

3.1 .1 Mean sequared error . . . . . . . . . . 33

3.1 .2 Simulation . . . . . . . . . 36

3.2 Current status model . . . . . . . . . . . . 43

3.2.1 Distribution of $X$ is known . . . . . . . . . 44

3.2.2 Model 1: $X \sim p(x)=I_{(0,1)}(x) \ldots . . . . .46$

3.2.3 Model 2: $X \sim p(x), p(x)$ is known . . . . . . 48

3.2.4 Model 3: $X \sim p(x)$, with $\operatorname{supp}\{p\}=\mathbb{R}_{+} \ldots 49$

3.2.5 Distribution of $X$ is unknown . . . . . . . 50

3.2 .6 Estimation . . . . . . . . . . 52

3.2 .7 Simulation .............. 56 
3.3 Length-biased model . . . . . . . . . . . . . 57

3.3.1 Mean Squared Error of $f_{\alpha, b}^{L B} \ldots \ldots . . . .58$

4 Conclusion and FutureWork 62

Bibliography 


\section{List of Tables}

2.1 Records of the Average $L_{2}$-errors, when $X \sim \operatorname{Gamma}(3,1 / 2)$

2.2 Comparison of Average $L_{2}$-errors of $\hat{f}_{\alpha, b}$ and the $\mathrm{KDE}$ $\hat{f}_{h}$ when $b=1.23 \ldots \ldots \ldots \ldots \ldots \ldots$

2.3 Records of the errors in sup-norm, when $X \sim \operatorname{Gamma}(2,1) 29$

3.1 Records of Average $L_{2}$-errors $d_{\alpha, b}\left(\times 10^{2}\right)$, when $X \sim$ $\log$-normal $(1,1)$ and $C \sim \operatorname{Gamma}(3,3) \ldots \ldots . \quad \ldots 9$

3.2 The Records of Average-errors in sup-norm for $\hat{f}_{h}$ and $\hat{f}_{\alpha, b}^{R C}$ when $b=1.13 \ldots \ldots \ldots \ldots \ldots \ldots$

3.3 Records the Average $L_{2}$-errors $\left(\times 10^{2}\right)$ of $\hat{f}_{\alpha, b}^{R C}$ (with $\alpha=30, b=1.13$ ) and $G$-estimate proposed in [3] . . 42

3.4 Records of errors in sup-norm for $F_{\alpha, T} \ldots \ldots \ldots \ldots$

3.5 Records of sup-norm-errors $d_{\alpha, b} \ldots \ldots \ldots \ldots \ldots$

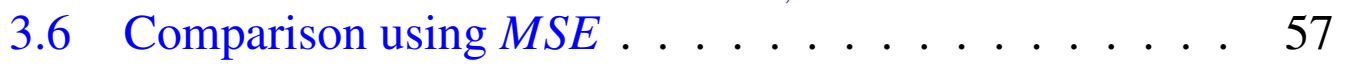

3.7 Records of errors in sup-norm $\ldots \ldots \ldots$. . . . . 61 


\section{List of Figures}

$2.1 \hat{f}_{\alpha, b}$ (dashed curve) and $f$ (solid curve) when $\alpha=80$ and $b=1.26 \ldots \ldots \ldots \ldots \ldots \ldots \ldots \ldots$

$2.2 \hat{f}_{\alpha, b}$ (dashed curve) and $f$ (solid curve) when $\alpha=80$ and $b=1.26, n=500 \ldots \ldots \ldots \ldots \ldots$

$2.3 \hat{f}_{\alpha, b}(x)$ curve (dashed), KDE $\hat{f}_{h}$ curve (dotted), and $f$ curve (solid) when $\alpha=100, b=1.26$, and $n=500 . ~ .24$

2.4 Approximation curve (blue dots) $m_{\alpha, b}(x)$ with $\alpha=100, b=$ 1.15 , the true $m(x)=\frac{x+2}{x+1}$ (orange dots). . . . . . 28

3.1 Estimated $\hat{f}_{\alpha, b}$ (dashed curve) with (a) $\alpha=40, b=$ 1.30 , (b) with $\alpha=80, b=1.13$, The true $f$ (solid curve).

3.2 Estimated $\hat{f}_{\alpha, b}^{R C}$ (orange) and the true function $f$ (blue) with $(b=1.30, \alpha=40), \mathrm{n}=500 . \ldots \ldots \ldots$

3.3 Linearly interpolated estimate $\hat{f}_{\alpha, b}^{R C}$ (orange), and the true function $f$ (blue) with $(b=1.30, \alpha=40), \mathrm{n}=500 . \quad 40$

3.4 Estimates $\hat{f}_{\alpha, b}^{R C}$ (dashed), $\hat{f}_{h}$ (dotted), true curve $f$ (solid) with $(b=1.10, \alpha=100) \ldots \ldots \ldots \ldots \ldots \ldots$

3.5 Estimation of $S$ (blue) by $\hat{S}_{\alpha, b}^{R C}$ (red) and $\hat{S}^{K M}$ (black) when $\alpha=80, b=1.55$, and $n=1000 \ldots \ldots .43$

3.6 Approximed $F_{\alpha, T}$ (orange curve) with $\alpha=80$, the true $F_{T}$ (black curve). . . . . . . . . . . . . 47

3.7 Approximed $F_{\alpha, T}$ (dashed curve ) with $\alpha=350$, the

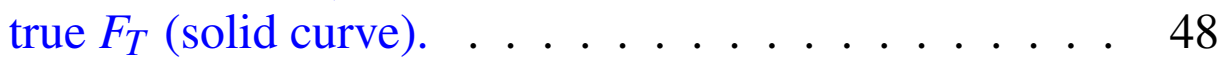


3.8 Approximed curve (orange) $F_{\alpha, T}$ with $\alpha=40, b=2.748$, the true $F_{T}$ (dashed black). . . . . . . . . 50

3.9 Approximion $F_{\alpha, T}$ (orange curve) with $\alpha=100$, the true $F_{T}$ (dotted curve). . . . . . . . . . . . 52

3.10 Estimation curve (orange) $F_{\alpha, T}$ with $\alpha=100$, the true $F_{T}$ (dotted blue). . . . . . . . . . . 56

3.11 Estimated $f_{\alpha, b}^{L B}$ (dashed curve) and the true $f$ (solid curve), when $\alpha=25, b=1.25$, and $n=1000 . \ldots 61$ 


\section{Chapter 1}

\section{Introduction}

Survival analysis is an important statistical topic in many areas such as engineering and medicine. Many statistical methods for data survival time have been presented and developed rapidly over the past few decades. The classical moment problem is the most important one. In this thesis we are intersted in estimating the unknown probability density function (pdf) and comulative distribution function (cdf) based on their sequence of moments and scaled Laplace transform. The scaled Laplace transform was suggested by Mnatsakanov [38]. The approximation rate of the inversion Laplace transform mentioned above is mainly used in the framework of the Hausdorff moment problem, when the target function $F$ has a compact support.

Mnatsakanov [38] modified the scaled version of the moment-recovered (MR) Laplace transform inversion that enables us to apply it in the case of the Stieltjes moment problem as well, i.e., when $T=\infty$. The 
moment-determinacy of probability distributions and their framework approximation of inverse moment problem are investigated in [34], [35] and [36].

This dissertation is intended to investigate the asymptotic properties of the density estimation in several statistical models. It is worth mentioning that the moments of underlying distribution are related in a simple manner to the moment of the sampled ones. Hence, the method of moments provides a relatively simple and alternative method of estimation in corresponding models. A rough classification according to the difficulty of indirect estimation problems. Let us outline several relationships between the moments of the underlying function and the sampled ones, see [37]. We assume that each of $F$ is determined uniquely in terms of its moments. According to the deffinition, if for two distributions $F$ and $G$ we have $\int x^{j} d F=\int x^{j} d G$ for all $j=1,2, \ldots$, then $F$ and $G$ coincide. In this case we say that the moment problem has a unique solution and $F$ is called M-determinate. The unique determinate has been studied in [43].

\subsection{Complete models}

An interesting problem in a direct model is estimating the pdf or cdf of a random variable $X$. There are several well known techniques for complete data, e.g. Lejeune and Sarda [29] worked on local linear esti- 
mator. Jones [12] and Jones and Forster [19] investigated the boundary kernel, Muller [39] studied the smooth optimum kernel, and Marron and Ruppert [31] used a transformation approach. To solve the boundary bias problem for the nonnegative data, Chen [11] considered the gamma kernel estimator. Jones [18] and Chen [11] showed that the local linear estimator achieves better results than the boundary kernel estimator of Muller [39]. Bouezmarni and Rolin [6] derived the exact asymptotic constants of uniform and $L_{1}$-errors for the kernel density estimator with Beta kernel. Moreover, recovering the cumulative distribution function of a positive random variable via the scaled Laplace transform has been studied by Mnatsakanov and Sarkisian [38]. We use the scaled Laplace transform $\mathcal{L}_{t}(f)=\int_{0}^{\infty} e^{-c t x} f(x) d x$ to estimate and derive the asymptotic properties of pdf.

Assuming that $X$ is a continuous random variable with survival function $S(x)$, the mean residual life time (MRLT) function $m(x)$ is defined by (see [26], [23], and [16])

$$
m(x)=E(X-x \mid X>x)=\frac{\int_{x}^{\infty} S(u) d u}{S(x)}
$$

The MRLT function, also known as the expected remaining life function. The MRLT function has been studied by various authors. Chaubey and Sen [12] introduced the smooth estimator of the mean residual life. The limiting properties of the mean residual life time have been studied 
by Meilijson [32] and Balkema and de Hann [3]. Some other literature proposed the nonparametric estimators of $m(x)$. For example, Ruiz and Guillamòn [40] estimated $\int_{x}^{\infty} S(u) d u$ in $m(x)$ by a recursive kernel estimate and used the empirical survival function to estimate $S(x)$. Belkacem and Alexandre [4] use the local linear fitting technique to estimate the mean residual life time function that depends on the classical kernel smoothing method. We introduce a new approximation to recover $m(x)$ by using the scaled Laplace transform $\mathcal{L}_{t}(F), t \in \mathbb{N}_{\alpha}, \alpha=0,1,2, .$. of lifetime variable $X$.

\subsection{Incomplete models}

The special cases of incomplete models that we concentrate on in this work are restricted to right censoring, current status, and weighted (length baised).

In the right censoring model, censoring occurs when we have some information about individual survival time, but sometimes we do not know the survival time exactly. In fact, we have $X \sim F, C \sim G$, and we only observe the pair $(Z, \delta)$, where $Z=\min (X, C)$, and $\delta=I\{X \leq C\}$, and $I$ is indicator function. In this construction our task is estimating the density $f=F^{\prime}$.

As a simple example of censoring, consider leukemia patients followed until they go out of remission. If for a given patient, the study 
ends while the patient is still in remission (i.e., doesn't get the event), then that patient's survival time is censored. We know that, for this person, the survival time is at least as long as the period that the person has been followed, but if the person goes out of remission after the study ends, we do not know the complete survival time. There are generally three reasons why censoring may occur: (1) a person does not experience the event before the study ends; (2) a person is lost to follow-up during the study of period; (3) a person withdraws from the study because of death (if the death is not the event of interest) or some other reason (e.g., adverse drug reaction or other competing risks)[27]. Kaplan and Meier [25] proposed a classical method for nonparametric models for right censoring survival function $\hat{S}(x)$. Since then the nonparametric methods had been applied extensively in statistical survival analysis of missing data.

A fixed symmetric kernel density with bounded support and bandwidth parameters is the most well known method for estimating the density function. The properties of this estimator have been studied by Földes et al.[13]; Mielniczuk [33] or Zhang [49] and others. The previous literature showed that the bandwidth has more impact than the kernel on the resulting estimate. Therefore, the order of bais of the standaerd kernel density estimator is larger near the endpoints. The problem of bias is also known as the edge effect. Bouezmarni, 
El Ghouch and Mesfioui [7] studied another estimator, based on the gamma kernels for the density function, that is free of bias. This estimator achieves the optimal rate of convergence in terms of integrated mean square error.

In current status data is also known as case 1 interval censroing. In this model the target function is the time of occurrence of some event, but observations are limited to indicators of whether or not the event has occurred at the examination time. Basically, let $T$ be a "life time" of some disease with unknown cumulative distribution function (cdf) $F_{T}$. In this model, we are not able to observe the survival time $T$. Instead, we observe the pair $(X, I\{T \leq X\})$. In epidemiology, these censoring schemes also arise for instance in AIDS studies or more generally in the study of infectious diseases when the infection time is an unobservable event. The asymptotic properties of estimators for nonparametric models with current status data have drawn considerable attention from statistical researchers. The first studies of nonparametric likelihood estimation (NPMLE) with current status data by Ayer et al.[1] Van Eeden [45] and [46]. Groeneboom [14], Groeneboom and Wellner [15], and Huang and Wellner [24] investigated the asymptotic properties of a nonparametric maximum likelihood estimator. Geer [44] showed the NPMLE of survival function converges at rate $n^{-1 / 3}$ in $L_{2}$-norm. Brunel and Comte [8] studied the projection methods to 
estimate cdf. In contrast to the NPMLE, Yang [48] proposed the locally linear smoother may not be monotone, but has a better convergence rate than the NPMLE when the density $f$ is smooth and the kernel function and bandwidth are properly chosen.

A special case of a weighted model is the length-biased model. The data of these models arises mostly from biomedicine, quality control or epidemiology. We are interested in estimating the pdf $f$ and $\operatorname{cdf} F$ of a positive random variable $X$, but we observe a random variable $Y$ with density $g(y)=\frac{w(y)}{W} f(y)$. Where the weight function $w$ and the total wieght $W=\int_{0}^{\infty} w(x) f(x) d x$ may not be known. Gill, Vardi, and Wellner [17] examine the large sample behavior of the NPMLE $F_{n}$ of the common underlying disribution $F$. The theoretical properties of kernel density estimation in the context of length-biased data was proposed by Borrajo et al. [9]. Vardi( [47]) derives the maximum likelihood estimator of the distribution function under weighted sampling and that the estimation of the mean is $\sqrt{n}$ consistent. Estimating a probability density function $f$ that is based on $g$, deriving the asymptotic normality and the rate of convergence in mean squared error (MSE) by using a sequence of moments proved by Mnatsakanov and Ruymgaart [37]. Jones [20] proposed a kernel density estimator for length biased data, and he showed that this estimator has better asymptotic properties. The multivariate case of this kernel density estimator was intorduced by 
Ahmad [2]. Two Fourier series based on kernel density estmation were investigated by Jones and Karunamuni[21].

\subsection{Moment recovery of density estimation}

In this work we assume that the probability density function defined

on $\mathbf{R}_{+}$is unique determinate by it's moment (M-determinate). The functions with conditions either M-determinate or M-indeterminate is a classical mathematical problem and investigated by many authors , see, for example,[28], [30], [41], and [42]. We consider two approximations of the Laplace transform inversions recovering cdf $F$ and its derivetive $f$. Where the cdf $F$ is considered to be an absolutely continuous function that has a support in $\mathbf{R}_{+}=[0, \infty)$. Let $f$ be its probability density function (pdf) with respect to the Lebesgue measure on $\mathbf{R}_{+}$. Suppose that a random variable $X$ is distributed according to $\mathrm{F}$.

Assume that we are given the sequence $\mathcal{L}(F)=\left\{\mathcal{L}_{t}(F), t \in \mathbb{N}_{\alpha}\right\}$ defined by the scaled Laplace transform of F:

$$
\mathcal{L}_{t}(F)=\int_{0}^{\infty} e^{-c t x} d F(x), \quad t \in \mathbb{N}_{\alpha}, \mathbb{N}_{\alpha}=\{0,1,2, \ldots, \alpha\} .
$$

To simplify the notation let us assume in (1.2) that the scale value $c=\ln (b)$ for some $1<b \leq \exp (1)$.

Let us denote the pdf of $\beta(p, q)$ distribution by

$$
\beta(u, p, q)=\frac{\Gamma(p+q)}{\Gamma(p) \Gamma(q)} u^{p-1}(1-u)^{q-1}, 0<u<1,
$$


where the shape parameters $p, q>0$, where $p=\left[\alpha b^{-x}\right]+1$ and $\alpha-$ $\left[\alpha b^{-x}\right]+1$, and let

$$
\beta_{\alpha, x}^{*}(\cdot)=\beta\left(\cdot,\left[\alpha b^{-x}\right]+1, \alpha-\left[\alpha b^{-x}\right]+1\right)
$$

with mean

$$
\eta_{\alpha}^{*}=\frac{[\alpha x]+1}{\alpha+2}
$$

and variance

$$
\sigma_{\alpha}^{*}=\frac{([\alpha x]+1)(\alpha-[\alpha x]+1)}{(\alpha+2)^{2}(\alpha+3)} .
$$

Consider the following approximations of $\operatorname{cdf} F$ and $\operatorname{pdf} f$, respectively, introduced in Mnatsakanov and Sarkisian [38].

For each $x \in \mathbf{R}_{+}$, define

$$
\begin{gathered}
\mathcal{K}_{\alpha}^{-1} \mathcal{L}(F)=1-\sum_{k=0}^{\left[\alpha b^{-x}\right]} \sum_{j=k}^{\alpha}\left(\begin{array}{l}
\alpha \\
j
\end{array}\right)\left(\begin{array}{l}
j \\
k
\end{array}\right)(-1)^{j-k} \mathcal{L}_{j}(F) \\
\left(\mathfrak{B}_{\alpha}^{-1} \mathcal{L}(F)\right)(x)=\frac{\ln b\left[\alpha b^{-x}\right]}{\alpha} \frac{\Gamma(\alpha+2)}{\Gamma\left(\left[\alpha b^{-x}\right]+1\right)} \sum_{m=0}^{\alpha-\left[\alpha b^{-x}\right]} \frac{(-1)^{m} \mathcal{L}_{\left(m+\left[\alpha b^{-x}\right]\right)}(F)}{\Gamma(m+1) \Gamma\left(\alpha-\left[\alpha b^{-x}\right]-m+1\right)}
\end{gathered}
$$

We suggest to use these formulas as a basic construction for estimation problem in the models that are mentioned below. 


\subsection{Organization of dissertation}

We organize this dissertation as follows. In Chapter 1 we introduced the methods and problems that will be discussed and investigated in this thesis. In Chapter 2, two complete models are considered: (a) direct model (b) Mean residual life time. The proposed construction of the distribution and density functions is based on the knowledge of the sequence of moments of scaled Laplace transform inversion $\mathcal{L}_{F}$ up to order $\alpha$. We derive the local asympototic properties of mean square error (MSE) of the underlying denstiy function of direct model.

Chapter 3 examines different kinds of the incomplete models such as: (a) right censoring (b) current status (c) length baised models. In the right censoring model, we extract the estimation of the density function with unbounded support based on its moments. We also explain the mean square error with of the density function and conduct a simulation study based on the average of $L_{2}$ error. Two cases of recovering distribution function are investigated in the current status model: (a) the distribution of the variable $X$ is known and (b) the distribution of $X$ is unknown. We investigate the upper bound for uniform rate as well as the MSE. In the length baised model we estimate the probability density function of unbounded support based on wieghted observation by using their sequence of moments. 
In all the chapters some simulation study and comparisons with other different costructions are conducted. Illustrations via graphs and tables with the values of errors of estimated functions are also provided. Furthermore, we studied the asymptotic behavior of the error between the estimated and theoretical functions.

The outlines and conclusions related to this work and some future work is explained in Chapter 4. 


\section{Chapter 2}

\section{Complete Model}

In this chapter we are going to implemant the applications of moments that were reviwed in chapter 1 . This implementaion was used in order to estimate the probability density function and derive the asympototic properties. Also, it was uesd to approximate the mean residual life function. The simulation study will be presented as well.

\subsection{Direct model}

Consider a sequence of independent and identically distributed (iid) random variables $X_{1}, X_{2}, \ldots, X_{n}$ with probability density function $f$. Assuming that $\operatorname{supp}\{f\}=\mathbf{R}_{+}$, we propose the following estimate of $f$ :

$$
\tilde{f}_{\alpha, b}(x)=\left(\mathfrak{B}_{\alpha}^{-1} \tilde{\mathcal{L}}_{F}(t)\right)(x), \quad x \in \mathbb{R}_{+},
$$

where $\tilde{\mathcal{L}}_{F}(t)$ is the estimated Laplace transform of $F$. For example, one can take

$$
\tilde{\mathcal{L}}_{F}(t)=\int_{\mathbf{R}_{+}} e^{-c t x} d \tilde{F}(x),
$$


with $\tilde{F}$ to be any consistent estimate of $F$. One such estimator is the empirical distribution function $\hat{F}_{n}$ of the sample $X_{1}, X_{2}, \ldots, X_{n}$ drawn from $F$. If such a sample is available, then after applying the normalizing factor $\alpha /(\alpha+1)$,we obtain the following estimate of $f$ :

our construction (1.4) will produce the estimate:

$$
\hat{f}_{\alpha, b}(x)=\frac{\alpha}{\alpha+1}\left(\mathfrak{B}_{\alpha}^{-1} \widehat{\mathcal{L}}(F)\right)(x), \quad x \in \mathbb{R}_{+} .
$$

Here

$$
\widehat{\mathcal{L}}_{t}(F)=\int_{\mathbf{R}_{+}} e^{-c t x} d \widehat{F}_{n}(x)=\frac{1}{n} \sum_{i=1}^{n} e^{-t c X_{i}},
$$

for some fixed $b>1$ and $c=\ln b$. The later construction can be rewritten in the following form:

$$
\begin{aligned}
\hat{f}_{\alpha, b}(x) & =\left(\frac{\alpha}{\alpha+1}\right) \frac{\left[\alpha b^{-x}\right] \ln (b) \Gamma(\alpha+2)}{\alpha \Gamma\left(\left[\alpha b^{-x}\right]+1\right)} \frac{1}{n} \sum_{i=1}^{n} \sum_{m=0}^{\alpha-\left[\alpha b^{-x}\right]} \frac{(-1)^{m} b^{-\left(m+\left[\alpha b^{-x}\right]\right) X_{i}}}{m !\left(\alpha-\left[\alpha b^{-x}\right]-m\right) !} \\
& =\frac{\left[\alpha b^{-x}\right] \ln (b) \Gamma(\alpha+2)}{(\alpha+1) \Gamma\left(\left[\alpha b^{-x}\right]+1\right)} \frac{1}{n} \sum_{i=1}^{n} \sum_{m=0}^{\alpha-\left[\alpha b^{-x}\right]} \frac{\left(-b^{-X_{i}}\right)^{m}\left(b^{\left.-X_{i}\right)}\left[\alpha b^{-x}\right]\right.}{m !\left(\alpha-\left[\alpha b^{-x}\right]-m\right) !} \\
& =\frac{\left[\alpha b^{-x}\right] \ln b}{\alpha+1} \frac{1}{n} \sum_{i=1}^{n} \beta\left(b^{-X_{i}},\left[\alpha b^{-x}\right]+1, \alpha-\left[\alpha b^{-x}\right]+1\right) \\
& =\frac{\left[\alpha b^{-x}\right] \ln b}{\alpha+1} \frac{1}{n} \sum_{i=1}^{n} \beta_{\alpha, x}^{*}\left(b^{-X_{i}}\right), \quad x \in \mathbb{R}_{+}
\end{aligned}
$$

\subsubsection{Mean squared error}

In this section we will study the local properties of estimator $f_{\alpha, b}(x)$. Without explicit reference, it will be assumed that the following conditions are satisfied. Namely, assume that the underlying density satisfies 
conditions:

$$
\begin{aligned}
& f \in C^{(2)}\left(\mathbf{R}_{+}\right) \quad \text { with } \quad C_{k, f}=\sup _{x \in[0, \infty)}\left|b^{k x} f^{\prime}(x)\right|<\infty, k=1,2, \\
& C_{3, f}=\sup _{x \in[0, \infty)}\left|b^{2 x} f^{\prime \prime}(x)\right|<\infty, \quad C_{4, f}=\sup _{x \in[0, \infty)}\left|b^{x} f(x)\right|<\infty, \\
& C_{5, f}=\sup _{x \in[0, \infty)}\left|b^{2 x} f(x)\right|<\infty
\end{aligned}
$$

The following theorem provides the upper bound for Mean Square Error (MSE) of $\hat{f}_{\alpha, b}$. Note that

$\operatorname{MSE}\left\{\hat{f}_{\alpha, b}(x)\right\}=E\left|\hat{f}_{\alpha, b}(x)-f(x)\right|^{2}:=\operatorname{var}\left\{\hat{f}_{\alpha, b}(x)\right\}+\left(E \hat{f}_{\alpha, b}(x)-f(x)\right)^{2}$

Theorem 1. If conditions (2.6) are satisfied, then for MSE of $\hat{f}_{\alpha, b}(x)$ we have:

$$
\begin{aligned}
\operatorname{MSE}\left\{\hat{f}_{\alpha, b}(x)\right\} & \leq n^{-4 / 5}\left[\left(\frac{2 b^{x}\left|f^{\prime}(x)\right|}{\ln b}+\frac{b^{2 x}\left|f^{\prime}(x)\right|}{2 \ln ^{2} b}+\frac{b^{x}\left|f^{\prime \prime}(x)\right|}{2 \ln b}\right)^{2}\right. \\
& \left.+\frac{f(x) \ln b}{\sqrt{\pi\left(b^{x}-1\right)}}\right]+o\left(n^{-4 / 5}\right)
\end{aligned}
$$

provided that we choose $\alpha=\alpha(n) \sim n^{2 / 5}, n \rightarrow \infty$.

The proof of Theorem 1 is based on investigating the asymptotic behavior of the bias and variance terms of the MSE of $\hat{f}_{\alpha, b}$ :

$\operatorname{MSE}\left\{\hat{f}_{\alpha, b}(x)\right\}:=E\left|\hat{f}_{\alpha, b}(x)-f(x)\right|^{2}=\operatorname{var}\left\{\hat{f}_{\alpha, b}(x)\right\}+\left(\operatorname{Bias}\left\{\hat{f}_{\alpha, b}(x)\right\}\right)^{2}$

where $\operatorname{Bias}\left\{\hat{f}_{\alpha, b}(x)\right\}=f_{\alpha, b}(x)-f(x)$ and $f_{\alpha, b}(x):=E\left(\hat{f}_{\alpha, b}(x)\right)$. 
Lemma 2. (i): $f_{\alpha, b}(x)$ converges uniformly to $f(x)$ as $\alpha \rightarrow \infty$, and for each $x>0$, the absolute value of the bias term of $\hat{f}_{\alpha, b}(x)$ is estimated from above as follows:

$\left|\operatorname{Bias}\left\{\hat{f}_{\alpha, b}(x)\right\}\right| \leq \frac{1}{\alpha+1}\left\{\frac{2 b^{x}\left|f^{\prime}(x)\right|}{\ln b}+\frac{b^{2 x}\left|f^{\prime}(x)\right|}{2 \ln ^{2} b}+\frac{b^{x}\left|f^{\prime \prime}(x)\right|}{2 \ln b}\right\}+o\left(\frac{1}{\alpha}\right)$

as $\alpha \rightarrow \infty$.

(ii): For each $x>0$, the asymptotic expression for variance of $\hat{f}_{\alpha, b}(x)$ we have

$$
\operatorname{var}\left\{\hat{f}_{\alpha, b}(x)\right\}=\frac{\sqrt{\alpha}}{n} \frac{f(x) \ln b}{\sqrt{\pi\left(b^{x}-1\right)}}+o\left(\frac{\sqrt{\alpha}}{n}\right),
$$

as $\sqrt{\alpha} / n \rightarrow 0, \alpha, n \rightarrow \infty$.

Proof of Lemma 2. Because $\hat{f}_{\alpha, b}=(\alpha /(\alpha+1)) \mathscr{B}_{\alpha}^{-1} \hat{\mathcal{L}}(F)$ has representation (2.5), we have

$$
\begin{aligned}
& E \hat{f}_{\alpha, b}(x)=\frac{\left[\alpha b^{-x}\right] \ln b}{\alpha+1} \frac{1}{n} \sum_{i=1}^{n} E \beta\left(b^{-X_{i}},\left[\alpha b^{-x}\right]+1, \alpha-\left[\alpha b^{-x}\right]+1\right) \\
& =\frac{\ln b \Gamma(\alpha+1)}{\Gamma\left(\left[\alpha b^{-x}\right]\right) \Gamma\left(\alpha+\left[\alpha b^{-x}\right]+1\right)} \int_{0}^{\infty}\left(b^{-u}\right)^{\left[\alpha b^{-x}\right]}\left(1-b^{-u}\right)^{\alpha-\left[\alpha b^{-x}\right]} f(u) d u .
\end{aligned}
$$


Change of variable under integral in (2.9) with $\tau=b^{-u}$ gives

$$
\begin{aligned}
& E \hat{f}_{\alpha, b}(x)=\frac{\ln b \Gamma(\alpha+1)}{\Gamma\left(\left[\alpha b^{-x}\right]\right) \Gamma\left(\alpha+\left[\alpha b^{-x}\right]+1\right)} \int_{0}^{1} \tau^{\left[\alpha b^{-x}\right]}(1-\tau)^{\alpha-\left[\alpha b^{-x}\right]} \frac{f\left(-\log _{b} \tau\right)}{\tau \ln b} d \tau \\
& =\frac{\Gamma(\alpha+1)}{\Gamma\left(\left[\alpha b^{-x}\right]\right) \Gamma\left(\alpha+\left[\alpha b^{-x}\right]+1\right)} \int_{0}^{1} \tau^{\left[\alpha b^{-x}\right]-1}(1-\tau)^{\alpha-\left[\alpha b^{-x}\right]} f\left(-\log _{b} \tau\right) d \tau \\
& =\int_{0}^{1} \beta\left(\tau,\left[\alpha b^{-x}\right], \alpha-\left[\alpha b^{-x}\right]+1\right) q(\tau) d \tau
\end{aligned}
$$

where $q=f \circ \phi$ denotes the composition of functions $f$ and $\phi(x)=$ $-\log _{b}(x)$. Therefore for expected value of $\hat{f}_{\alpha, b}$ we have

$$
f_{\alpha, b}(x)=E \hat{f}_{\alpha, b}(x)=\int_{0}^{1} \beta_{\alpha, x}(\tau) q(\tau) d \tau, q(\tau)=f\left(-\log _{b} \tau\right) .
$$

Here, $\beta_{\alpha, x}(\cdot):=\beta\left(\cdot,\left[\alpha b^{-x}\right], \alpha-\left[\alpha b^{-x}\right]+1\right)$. To complete the proof of Lemma $1(i)$ one can proceed in a similar way as it is done in Mnatsakanov et al. (2015). Namely, let us mention that the first and second derivatives of $q$ with respect to $\tau$ can be written as

$$
\begin{gathered}
q^{\prime}(\tau)=\left(f^{\prime} \circ \phi\right)(\tau) \phi^{\prime}(\tau) \\
q^{\prime \prime}(\tau)=\left(f^{\prime \prime} \circ \phi\right)(\tau) \phi^{\prime \prime}(\tau)+\left(f^{\prime} \circ \phi\right)(\tau) \phi^{\prime \prime}(\tau) .
\end{gathered}
$$

Evaluation of $q^{\prime}$ and $q^{\prime \prime}$ at $\tau=b^{-x}$ gives: $q^{\prime}\left(b^{-x}\right)=\frac{b^{x} f^{\prime}(x)}{\ln b}$ and $q^{\prime \prime}\left(b^{-x}\right)=$ $\frac{b^{2 x} f^{\prime}(x)}{\ln ^{2} b}+\frac{b^{x} f^{\prime \prime}(x)}{\ln b}$. Now, note that the sequence $\left\{\beta_{\alpha, x}(\cdot), \alpha=1,2, \ldots\right\}$ represents the sequence of $\delta$-functions with the mean and variance specified as follows:

$$
\eta_{\alpha}:=\int_{0}^{1} \tau \beta_{\alpha, x}(\tau) d \tau=\frac{\left[\alpha b^{-x}\right]}{\alpha+1}
$$




$$
\sigma_{\alpha}^{2}:=\int_{0}^{1}\left(\tau-\eta_{\alpha}\right)^{2} \beta_{\alpha, x}(\tau) d \tau=\frac{\left[\alpha b^{-x}\right]\left(\alpha-\left[\alpha b^{-x}\right]+1\right)}{(\alpha+1)^{2}(\alpha+2)}<\frac{1}{\alpha+1}
$$

and

$$
\left|\eta_{\alpha}-b^{-x}\right| \leq \frac{2}{\alpha+1}
$$

To derive the asymptotic form of the $\operatorname{Bias}\left\{\hat{f}_{\alpha, b}(x)\right\}=f_{\alpha, b}(x)-f(x)$ let us write

$$
f_{\alpha, b}(x)-f(x)=\int_{0}^{1} \beta_{\alpha, x}(\tau)\left\{q(\tau)-q\left(b^{-x}\right)\right\} d \tau .
$$

Applying the Taylor expansion of $q(\tau)$ around $\tau=b^{-x}$ we get:

$\operatorname{Bias}\left\{\hat{f}_{\alpha, b}(x)\right\}=\int_{0}^{1} \beta_{\alpha, x}(\tau)\left\{\left(\tau-b^{-x}\right) q^{\prime}\left(b^{-x}\right)+\frac{1}{2}\left(\tau-b^{-x}\right)^{2} q^{\prime \prime}(\tilde{\tau})\right\} d \tau$

Now adding and subtracting $\eta_{\alpha}$ from the first and second terms in the right hand side of (2.18) we obtain the upper bound for the absolute value of $\operatorname{Bias}\left\{\hat{f}_{\alpha, b}(x)\right\}$

$$
\begin{aligned}
& \mid \int_{0}^{1} \beta_{\alpha, x}(\tau)\left(\tau-\eta_{\alpha}\right) q^{\prime}\left(b^{-x}\right) d \tau+\left(\eta_{\alpha}-b^{-x}\right) q^{\prime}\left(b^{-x}\right) \int_{0}^{1} \beta_{\alpha, x}(\tau) d \tau \\
& +\frac{1}{2} \int_{0}^{1} \beta_{\alpha, x}(\tau)\left(\tau-\eta_{\alpha}\right)^{2} q^{\prime \prime}(\tilde{\tau}) d \tau+\frac{1}{2}\left(\eta_{\alpha}-b^{-x}\right)^{2} \int_{0}^{1} \beta_{\alpha, x}(\tau) q^{\prime \prime}(\tilde{\tau}) d \tau \mid \\
& \leq\left|\eta_{\alpha}-b^{-x}\right|\left|q^{\prime}\left(b^{-x}\right)\right|+\frac{1}{2} \sigma_{\alpha}^{2}\left\|q^{\prime \prime}\right\|+\frac{1}{2}\left(\eta_{\alpha}-b^{-x}\right)^{2}\left\|q^{\prime \prime}\right\|
\end{aligned}
$$

Now taking into account the bounds for $\sigma_{\alpha}^{2}$ and $\left|\eta_{\alpha}-b^{-x}\right|$ mentioned 
in (2.15) and (2.16), respectively, we obtain for the bias of $\hat{f}_{\alpha, b}$ : $\left|\operatorname{Bias}\left\{\hat{f}_{\alpha, b}(x)\right\}\right| \leq \frac{1}{\alpha+1}\left\{\frac{2 b^{x}\left|f^{\prime}(x)\right|}{\ln b}+\frac{b^{2 x}\left|f^{\prime}(x)\right|}{2 \ln ^{2} b}+\frac{b^{x}\left|f^{\prime \prime}(x)\right|}{2 \ln b}\right\}+o\left(\frac{1}{\alpha}\right)$

as $\alpha \rightarrow \infty$. The uniform convergence of $f_{\alpha, b}$ to $f$ follows from (2.20) and conditions (2.6).

Now, consider the variance of $\hat{f}_{\alpha, b}$. Taking into account (2.5) and (2.11), we obtain:

$$
\begin{aligned}
\operatorname{var}\left\{\hat{f}_{\alpha, b}(x)\right\} & =\frac{1}{n}\left\{\frac{\left[\alpha b^{-x}\right] \ln b}{\alpha+1}\right\}^{2} \operatorname{var}\left\{\beta_{\alpha, x}^{*}\left(b^{-X_{1}}\right)\right\} \\
= & \frac{1}{n}\left\{\frac{\left[\alpha b^{-x}\right] \ln b}{\alpha+1}\right\}^{2} E \beta_{\alpha, x}^{* 2}\left(b^{-X_{i}}\right)-\frac{1}{n}\left\{\frac{\left[\alpha b^{-x}\right] \ln b}{\alpha+1}\right\}^{2}\left(E \beta_{\alpha, x}^{*}\left(b^{-X}\right)\right)^{2} .
\end{aligned}
$$

At first let us investigate the asymptotic behavior of the first term in (2.21). We have

$\frac{1}{n}\left\{\frac{\left[\alpha b^{-x}\right] \ln b}{\alpha+1}\right\}^{2} E \beta_{\alpha, x}^{* 2}\left(b^{-X_{i}}\right)=\frac{1}{n}\left\{\frac{\left[\alpha b^{-x}\right] \ln b}{\alpha+1}\right\}^{2} \int_{0}^{\infty} \beta_{\alpha, x}^{* 2}\left(b^{-u}\right) f(u) d u$ $=\frac{1}{n}\left(\frac{\ln b \Gamma(\alpha+1)}{\Gamma\left(\left[\alpha b^{-x}\right]\right) \Gamma\left(\alpha-\left[\alpha b^{-x}\right]+1\right)}\right)^{2} \int_{0}^{\infty}\left(b^{-u}\right)^{2\left[\alpha b^{-x}\right]}\left(1-b^{-u}\right)^{2 \alpha-2\left[\alpha b^{-x}\right]} f(u) d u$ $=\frac{1}{n}\left(\frac{\ln b \Gamma(\alpha+1)}{\Gamma\left(\left[\alpha b^{-x}\right]\right) \Gamma\left(\alpha-\left[\alpha b^{-x}\right]+1\right)}\right)^{2} \int_{0}^{1} \tau^{2\left[\alpha b^{-x}\right]-1}(1-\tau)^{2 \alpha-2\left[\alpha b^{-x}\right]} q(\tau) \frac{d \tau}{\ln b}$ $=\xi_{\alpha}(x) \int_{0}^{1} \beta\left(\tau, 2\left[\alpha b^{-x}\right], 2 \alpha-2\left[\alpha b^{-x}\right]+1\right) q(\tau) d \tau$. 
Here

$\xi_{\alpha}(x)=\frac{\ln b}{n}\left(\frac{\Gamma(\alpha+1)}{\Gamma\left(\left[\alpha b^{-x}\right]\right) \Gamma\left(\alpha-\left[\alpha b^{-x}\right]+1\right)}\right)^{2} \frac{\Gamma\left(2\left[\alpha b^{-x}\right]\right) \Gamma\left(2 \alpha-2\left[\alpha b^{-x}\right]+1\right)}{\Gamma(2 \alpha+1)}$.

and according to Lemma $2(i)$ :

$$
\int_{0}^{1} \beta\left(\tau, 2\left[\alpha b^{-x}\right], 2 \alpha-2\left[\alpha b^{-x}\right]+1\right) q(\tau) d \tau \rightarrow f(x) \text { as } \quad \alpha \rightarrow \infty,
$$

uniformly with the rate of $1 / \alpha$. The order of magnitude of $\xi_{\alpha}(x)$ in (2.25) is specified as follows: for each $x>0$ we have

$$
\xi_{\alpha}(x) \sim \frac{\ln b}{\sqrt{\pi}} \frac{\alpha^{1 / 2}}{n}\left(\frac{b^{-x}}{1-b^{-x}}\right)^{1 / 2}, \alpha, n \rightarrow \infty .
$$

Hence, for the first term in the right-hand side of (2.21) we can write:

$$
\frac{1}{n}\left\{\frac{\left[\alpha b^{-x}\right] \ln b}{\alpha+1}\right\}^{2} E \beta_{\alpha, x}^{* 2}\left(b^{-X_{1}}\right)=\frac{\sqrt{\alpha}}{n} \frac{f(x) \ln b}{\sqrt{\pi\left(b^{x}-1\right)}}+o\left(\frac{\sqrt{\alpha}}{n}\right),
$$

as $\alpha, n \rightarrow \infty$. Consider the second term of (2.21): 


$$
\begin{aligned}
& \frac{1}{n}\left\{\frac{\left[\alpha b^{-x}\right] \ln b}{\alpha+1}\right\}^{2}\left(E \beta_{\alpha, x}^{*}\left(b^{-X}\right)\right)^{2}=\frac{1}{n}\left\{\frac{\left[\alpha b^{-x}\right] \ln b}{\alpha+1}\right\}^{2} \\
& \times\left(\frac{\Gamma(\alpha+2)}{\Gamma\left(\left[\alpha b^{-x}\right]+1\right) \Gamma\left(\alpha-\left[\alpha b^{-x}\right]+1\right)}\right)^{2} \\
& \times\left(\int_{0}^{\infty}\left(b^{-u}\right)^{\left[\alpha b^{-x}\right]}\left(1-b^{-u}\right)^{\alpha-\left[\alpha b^{-x}\right]} f(u) d u\right)^{2} \\
& =\frac{1}{n}\left(\frac{\ln b \Gamma(\alpha+1)}{\Gamma\left(\left[\alpha b^{-x}\right]\right) \Gamma\left(\alpha-\left[\alpha b^{-x}\right]+1\right)}\right)^{2}\left(\int_{0}^{1} \tau^{\left[\alpha b^{-x}\right]-1}(1-\tau)^{\alpha-\left[\alpha b^{-x}\right]} q(\tau) \frac{d \tau}{\ln (b)}\right)^{2} \\
& =\frac{1}{n}\left(\int_{0}^{1} \beta_{\alpha, x}(\tau) q(\tau) d \tau\right)^{2}=\frac{1}{n}\left(f(x)+O\left(\frac{1}{\alpha}\right)\right)^{2}, \text { as } \alpha \rightarrow \infty \text {. }
\end{aligned}
$$

Finally, from (2.21), (2.24), and (2.25) we obtain

$$
\operatorname{var}\left\{\hat{f}_{\alpha, b}(x)\right\}=\frac{\sqrt{\alpha}}{n} \frac{f(x) \ln b}{\sqrt{\pi\left(b^{x}-1\right)}}+o\left(\frac{\sqrt{\alpha}}{n}\right),
$$

as $\sqrt{\alpha} / n \rightarrow 0, \alpha, n \rightarrow \infty$.

Proof of Theorem 1. Combining the statements $(i)$ and (ii) of Lemma

2 we obtain:

$$
\begin{aligned}
\operatorname{MSE}\left\{\hat{f}_{\alpha, b}(x)\right\} & \leq \frac{1}{(\alpha+1)^{2}}\left\{\frac{2 b^{x}\left|f^{\prime}(x)\right|}{\ln b}+\frac{b^{2 x}\left|f^{\prime}(x)\right|}{2 \ln ^{2} b}+\frac{b^{x}\left|f^{\prime \prime}(x)\right|}{2 \ln b}\right\}^{2} \\
& +\frac{\sqrt{\alpha}}{n} \frac{f(x) \ln b}{\sqrt{\pi\left(b^{x}-1\right)}}+o\left(\frac{\sqrt{\alpha}}{n}\right) .
\end{aligned}
$$

Finally, taking $\alpha \sim n^{2 / 5}$ in (2.27), we arrive at (2.8). 


\subsubsection{Simulation}

In this section we simulated the underlying density function of the design which variable is chosen to be $X \sim \operatorname{Gamma}(a, b)$. We have generated $n=800$ independent random sample of $X_{i}$ 's from Gamma distribution with $a=3$ and $b=1 / 2$ to estimate the density function of the direct model $f_{\alpha, b}$. The Figure 2.1 displays the estimated density function when $\alpha=80$ and $b=1.26$, and the sample size $n=500$.

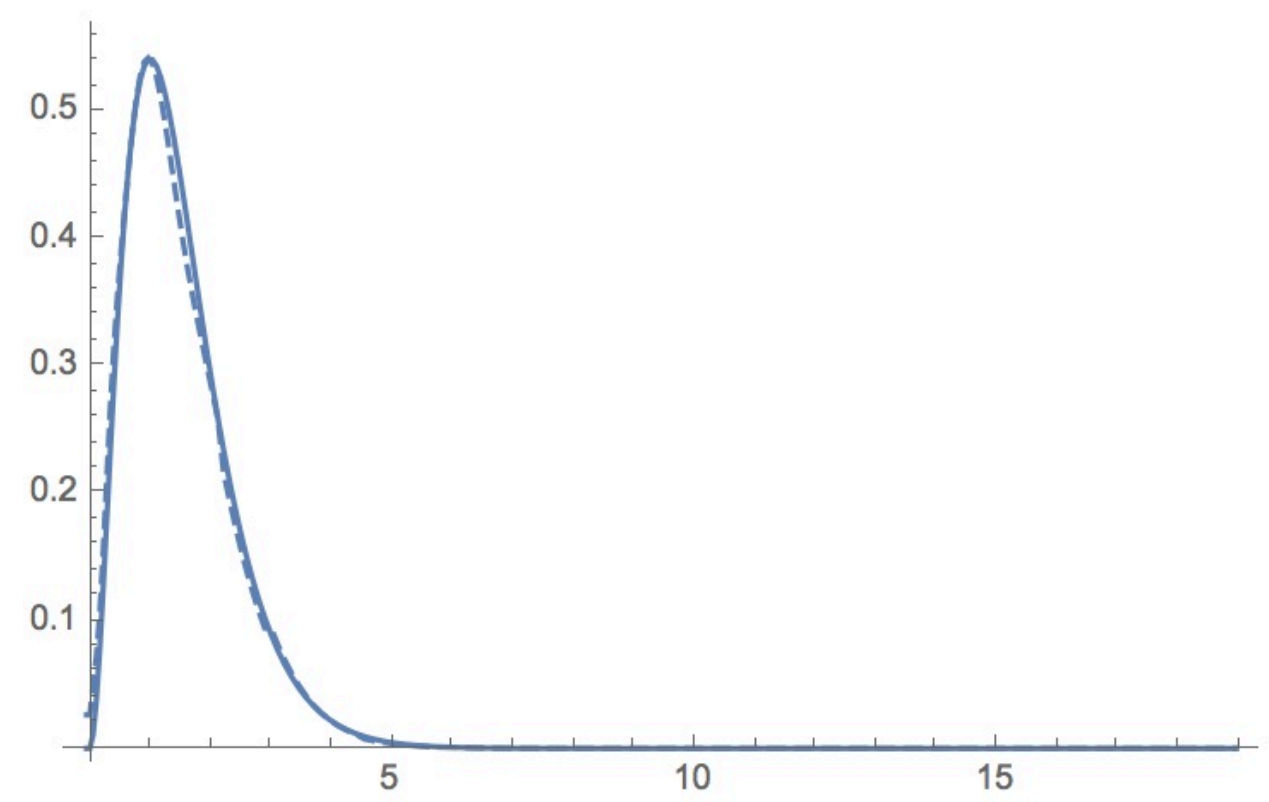

Figure 2.1: $\hat{f}_{\alpha, b}$ (dashed curve) and $f$ (solid curve) when $\alpha=80$ and $b=1.26$.

In addition, the Average $L_{2}$-errors are computed for different values of $\alpha, b$, and $n$, as it shown in Table 3.1.

From this table we identify the optimal values of $b$ as follows: $b \in$ $(1.23,1.29)$ when $\alpha=80,100$, and 120 , respectively. Figure 2.2 shows 
Table 2.1: Records of the Average $L_{2}$-errors, when $X \sim \operatorname{Gamma}(3,1 / 2)$

\begin{tabular}{|c||c|c|c||c|c|c|c|c|c|}
\hline \multicolumn{1}{|c|}{$d_{\alpha, b}$} & \multicolumn{3}{|c|}{$\mathrm{n}=300$} & \multicolumn{3}{c|}{$\mathrm{n}=500$} & \multicolumn{3}{c|}{$\mathrm{n}=800$} \\
\hline $\mathrm{b}$ & $\alpha=80$ & $\alpha=100$ & $\alpha=120$ & $\alpha=80$ & $\alpha=100$ & $\alpha=120$ & $\alpha=80$ & $\alpha=100$ & $\alpha=120$ \\
\hline 1.05 & 0.154 & 0.1153 & 0.0969 & 0.1492 & 0.1213 & 0.0998 & 0.1507 & 0.1209 & 0.0948 \\
1.15 & 0.0404 & 0.0294 & 0.0227 & 0.0397 & 0.0257 & 0.0179 & 0.0404 & 0.0269 & 0.0196 \\
1.21 & 0.0235 & 0.0158 & 0.0125 & 0.0219 & 0.0154 & 0.0084 & 0.0244 & 0.0154 & 0.0119 \\
1.23 & 0.0219 & 0.0140 & 0.0104 & 0.0217 & 0.0134 & 0.0090 & 0.0232 & 0.0147 & 0.0081 \\
1.25 & 0.0167 & 0.0111 & 0.0091 & 0.0166 & 0.0105 & 0.0092 & 0.0177 & 0.0109 & 0.0127 \\
1.26 & 0.0147 & 0.0104 & 0.0087 & 0.0175 & 0.0103 & 0.0120 & 0.0158 & 0.0111 & 0.0101 \\
1.27 & 0.0156 & 00103 & 0.0082 & 0.0145 & 0.0103 & 0.0104 & 0.0168 & 0.0096 & 0.0119 \\
1.28 & 0.0144 & 0.0099 & 0.0093 & 0.0144 & 0.0099 & 0.0102 & 0.015 & 0.0094 & 0.0117 \\
1.29 & 0.0139 & 0.0101 & 0.0136 & 0.0159 & 0.0101 & 0.0122 & 0.0158 & 0.0269 & 0.0095 \\
1.30 & 0.013 & 0.0984 & 0.0107 & 0.0123 & 0.0098 & 0.0106 & 0.0137 & 0.0095 & 0.0099 \\
1.50 & 0.0097 & 0.0063 & 0.0061 & 0.0089 & 0.0063 & 0.0064 & 0.0106 & 0.00752 & 0.00590 \\
1.80 & 0.0079 & 0.0039 & 0.0055 & 0.0073 & 0.0039 & 0.0058 & 0.0068 & 0.0042 & 0.0025 \\
1.90 & 0.0079 & 0.0049 & 0.0069 & 0.0057 & 0.0049 & 0.0021 & 0.0053 & 0.0037 & 0.0017 \\
2.0 & 0.0058 & 0.0071 & 0.0052 & 0.0051 & 0.0071 & 0.0039 & 0.0051 & 0.0039 & 0.0030 \\
2.01 & 0.0054 & 0.0046 & 0.0022 & 0.0066 & 0.0046 & 0.0031 & 0.0054 & 0.0040 & 0.0047 \\
\hline
\end{tabular}

the optimal $\alpha$ and $b$ when $X \sim \operatorname{Exponential}(2 / 3), \mathrm{n}=500$. 


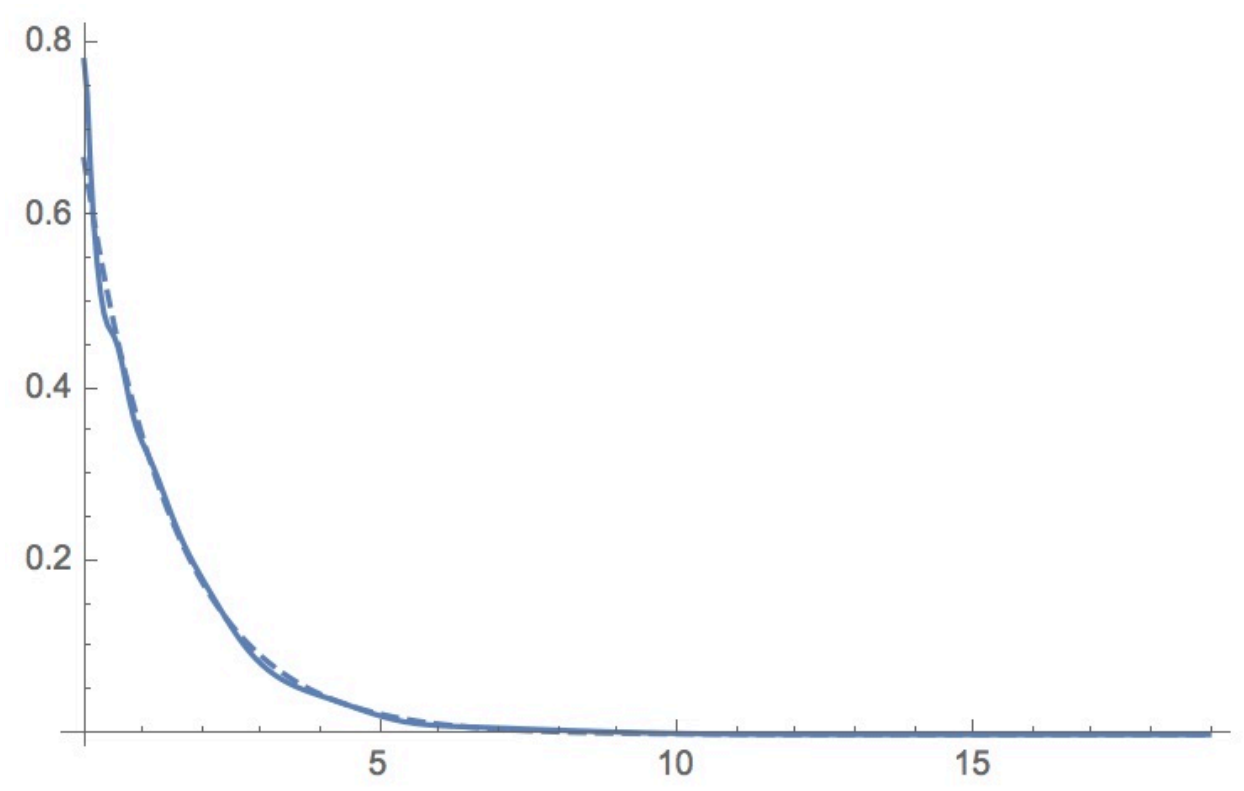

Figure 2.2: $\hat{f}_{\alpha, b}$ (dashed curve) and $f$ (solid curve) when $\alpha=80$ and $b=1.26, n=$ 500 .

Now let us compare the expressions of the main terms of MSE for $\hat{f}_{\alpha, b}(x)$ and the $\operatorname{KDE} \hat{f}_{h}(x)$.

For the direct model where $X_{i} \sim \operatorname{Gamma}(3,1 / 2)$, and compared with kernel density function using Gaussian and $h=n^{-1 / 5} \times \hat{\sigma} \times 1.06$ where $\mathrm{h}$ is the bandwidth and $\hat{\sigma}$ is the standard deviation of the sample. 


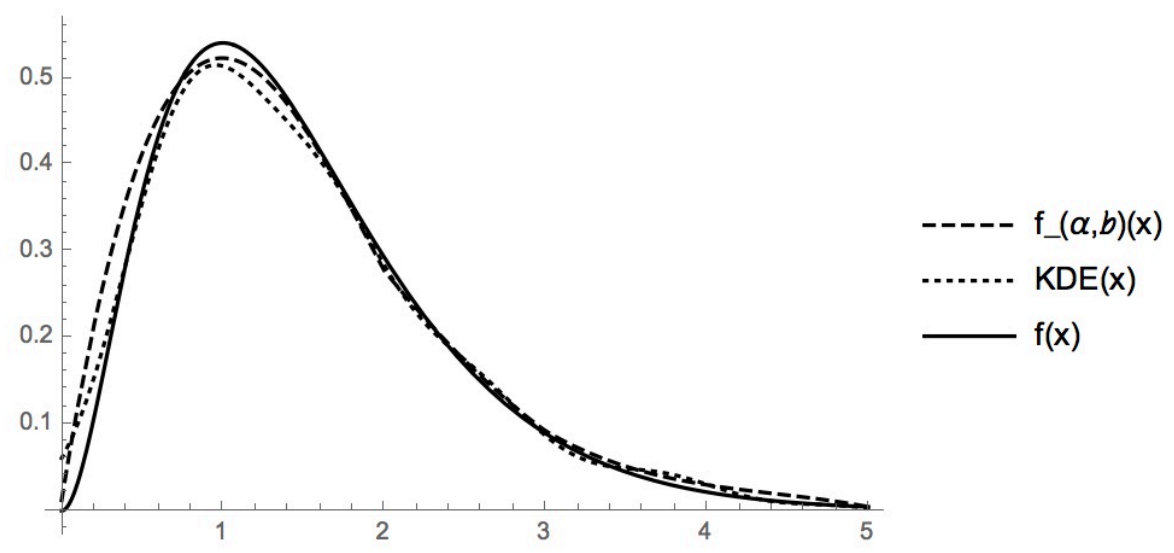

Figure 2.3: $\hat{f}_{\alpha, b}(x)$ curve (dashed), KDE $\hat{f}_{h}$ curve (dotted), and $f$ curve (solid) when $\alpha=100, b=1.26$, and $n=500$.

Table 2.2: Comparison of Average $L_{2}$-errors of $\hat{f}_{\alpha, b}$ and the KDE $\hat{f}_{h}$ when $b=1.23$

\begin{tabular}{|c|c|c|c|c|c|}
\hline \hline $\mathrm{n}$ & $\hat{f}_{80}$ & $\hat{f}_{100}$ & $\hat{f}_{120}$ & $f_{h}$ & $h$ \\
\hline 300 & 0.01004 & 0.0097 & 0.01071 & 0.01108 & 0.289 \\
500 & 0.0084 & 0.0088 & 0.0083 & 0.0088 & 0.263 \\
\hline
\end{tabular}

From Table 3.2 we conclude that the performances of $\hat{f}_{\alpha, b}$ when $b=$ 1.23 , and the KDE $\hat{f}_{h}$ (with $h=0.289,0.263$ ) are similar to each other when $X \sim \operatorname{Gamma}(3,1 / 2)$.

\subsection{Mean residual life time}

In this section we consider the problem of approximating the mean residual life function (mrlf) using the scaled Laplace transform defined in (1.2). The mrlf of lifetime variable $X$ with finite expectation is de- 
fined as :

$$
m(x)=E(X-x \mid X>x)=\frac{\int_{x}^{\infty} S(t) d t}{S(x)}:=\frac{\bar{Q}(x)}{S(x)} .
$$

To approximate $m(x)$ let us first approximate the numerator $\bar{Q}(x)$ using the scaled Laplace transform:

$$
\mathcal{L}_{t}(Q)=\int_{0}^{\infty} e^{-c t x} d Q(x)
$$

After integration by part in (2.29) we arrive at:

$$
\mathcal{L}_{t}(Q)=\frac{1}{t \ln b}\left(1-\mathcal{L}_{t}(f)\right),
$$

where $\mathcal{L}_{t}(f)=\int_{0}^{\infty} e^{-c t x} f(x) d x$. The following approximation of $\bar{Q}$ is suggested:

$$
\bar{Q}_{\alpha, b}(x)=\left(\mathcal{K}_{\alpha}^{-1} \mathcal{L}(Q)\right)(x)
$$

with

$$
\left(\mathcal{K}_{\alpha}^{-1} \mathcal{L}(Q)\right)(x)=\sum_{k=0}^{\left[\alpha b^{-x}\right]} \sum_{j=k}^{\alpha}\left(\begin{array}{l}
\alpha \\
j
\end{array}\right)\left(\begin{array}{l}
j \\
k
\end{array}\right)(-1)^{j-k} \mathcal{L}_{t}(Q) .
$$

The denominator of (2.28) can be approximated and estimated by:

$$
S_{\alpha, b}(x)=\left(\mathcal{K}_{\alpha}^{-1} \mathcal{L}(f)\right)(x) \text { and } \hat{S}_{\alpha, b}(x)=\left(\mathcal{K}_{\alpha}^{-1} \hat{\mathcal{L}}(f)\right)(x),
$$

respectively. Finally, we arrive at the following approximated and estimated versions of $m(x)$ :

$$
m_{\alpha, b}(x)=\frac{\bar{Q}_{\alpha, b}(x)}{S_{\alpha, b}(x)} \text { and } \hat{m}_{\alpha, b}(x)=\frac{\hat{\bar{Q}}_{\alpha, b}(x)}{\hat{S}_{\alpha, b}(x)},
$$


respectively. Here $\hat{\bar{Q}}_{\alpha, b}=\mathcal{K}_{\alpha}^{-1} \hat{\mathcal{L}}(\bar{Q})$.

Now, let us assume that $\operatorname{supp}\{f\}=\mathbf{R}_{+}$, and the follwing conditions are satisfied:

$$
\begin{aligned}
& C_{k, S}=\sup _{0<x<\infty}\left|b^{k x} S(x)\right|<\infty, \quad k=1,2 \\
& C_{3, S}=\sup _{0<x<\infty}\left|b^{2 x} S^{\prime}(x)\right|<\infty
\end{aligned}
$$

In the following theorem the upper bound of $m_{\alpha, b}(x)$ is derived.

Theorem 3. If functions $f$ and $f^{\prime}$ are bounded, the conditions (2.6) and (2.35) are satisfied, and $S(x)>0$ for each $x>0$, then

$$
\left|m_{\alpha, b}(x)-m(x)\right| \leq \frac{1}{S(x)-\frac{C_{f}}{\alpha}}\left(\frac{C_{S}}{\alpha+1}+\frac{m(0) C_{f}}{(\alpha+1) S(x)}\right)+o\left(\frac{1}{\alpha+1}\right),
$$

as $\alpha \rightarrow \infty$.

\section{Proof}

For each $x>0$ we can write the following inequality:

$$
\begin{gathered}
\left|\frac{\bar{Q}_{\alpha, b}(x)}{S_{\alpha, b}(x)}-\frac{\bar{Q}(x)}{S(x)}+\frac{\bar{Q}(x)}{S_{\alpha, b}(x)}-\frac{\bar{Q}(x)}{S_{\alpha, b}(x)}\right|=\left|\frac{\bar{Q}_{\alpha, b}(x)-\bar{Q}(x)}{S_{\alpha, b}(x)}+\frac{\bar{Q}(x)\left(S(x)-S_{\alpha, b}(x)\right)}{S(x) S_{\alpha, b}(x)}\right| \\
\leq \frac{1}{\left|S_{\alpha, b}(x)\right|}\left|\bar{Q}_{\alpha, b}(x)-\bar{Q}(x)\right|+\left|\bar{Q}(x) \frac{S_{\alpha, b}(x)-S(x)}{S_{\alpha, b}(x) S(x)}\right| \\
\leq \frac{1}{\left|S_{\alpha, b}(x)\right|}\left(\left|\bar{Q}_{\alpha, b}(x)-\bar{Q}(x)\right|+\frac{\bar{Q}(x)}{S(x)}\left|S_{\alpha, b}(x)-S(x)\right|\right) .
\end{gathered}
$$


Mnatsakanov and Sarkisian in [38] derived the uniform upper bound for $\left|S_{\alpha, b}(x)-S(x)\right|$ :

$$
\left|S_{\alpha, b}(x)-S(x)\right| \leq \frac{1}{\alpha+1}\left\{\frac{C_{4, f}}{\ln ^{2} b}+\frac{C_{5, f}}{2 \ln b}+\frac{C_{2, f}}{2 \ln ^{2} b}\right\}+o\left(\frac{1}{\alpha}\right)
$$

To estimate $\left|\bar{Q}_{\alpha, b}(x)-\bar{Q}(x)\right|$ we can apply similar steps used in Lemma 2 and obtain:

$$
\begin{aligned}
\left|\bar{Q}_{\alpha, b}(x)-\bar{Q}(x)\right| & \leq \frac{\alpha}{(\alpha+1)^{2}} \frac{C_{1, S}}{\ln b}+\frac{\alpha(\alpha+2)}{2(\alpha+1)^{3}}\left(\frac{C_{2, S}}{\ln ^{2} b}+\frac{C_{3, S}}{\ln b}\right) \\
+ & o\left(\frac{1}{\alpha}\right) .
\end{aligned}
$$

On the other hand, for sufficiently large values of $\alpha$ we have $\mid S_{\alpha, b}(x)-$ $S(x) \mid \leq \frac{C_{f}}{\alpha}$, where $C_{f}$ is the constant from the hand right side of (2.38). Hence, $S_{\alpha, b}(x)>S(x)-\frac{C_{f}}{\alpha}$. Finally, note that the function $\bar{Q}(x)$ is bounded by $\int_{x}^{\infty} S(t) d t \leq \int_{0}^{\infty} S(t) d t=m(0)$. 
Example 1. Let us consider the model with $X \sim \operatorname{gamma}(2,1)$. Corresponding survival function of $X$ has the form $S(x)=x e^{-x}+e^{-x}$. The Figure 2.4 displays $m_{\alpha, b}(x)$ evaluated with optimal values of $\alpha$ and $b$ specified by the records from Table 2.3.

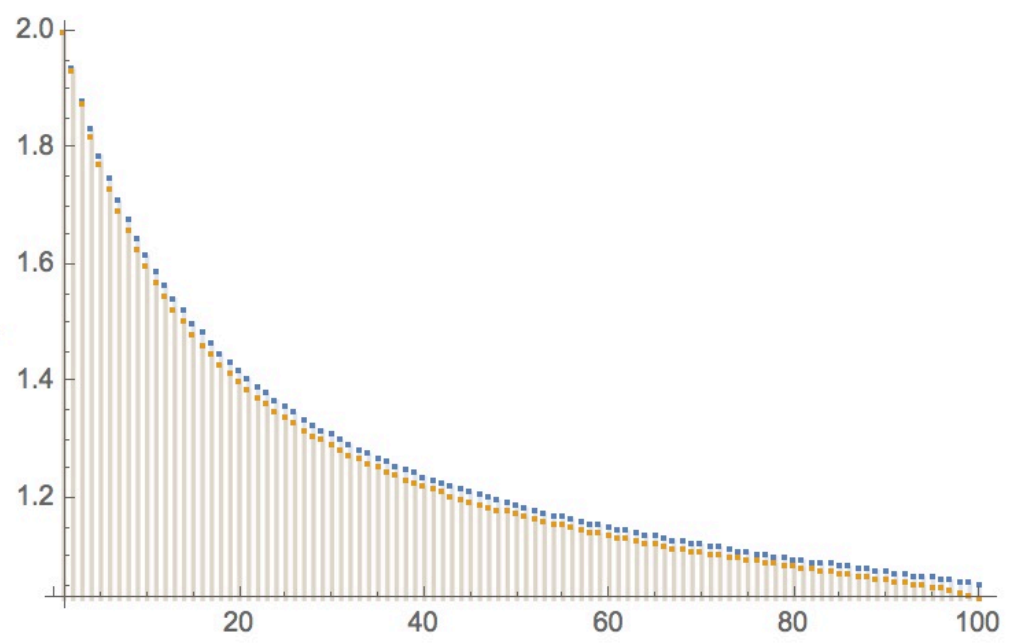

Figure 2.4: Approximation curve (blue dots) $m_{\alpha, b}(x)$ with $\alpha=100, b=1.15$, the true $m(x)=\frac{x+2}{x+1}$ (orange dots).

Records of errors in sup-norm of approximation for $\hat{m}_{\alpha, b}$ are shown in the table below. From these records we specify the smallest error that corresponds to values of $\alpha=100$ and $b=1.15$. 
Table 2.3: Records of the errors in sup-norm, when $X \sim \operatorname{Gamma}(2,1)$

\begin{tabular}{|c|c|c|c|c|c|}
\hline \multicolumn{7}{|c|}{$d_{\alpha, b}$} \\
\hline $\mathrm{b}$ & $\alpha=20$ & $\alpha=25$ & $\alpha=30$ & $\alpha=80$ & $\alpha=100$ \\
\hline 1.05 & 0.1773 & 0.1541 & 0.1352 & 0.0959 & 0.0487 \\
1.15 & 0.0871 & 0.0719 & 0.0613 & 0.0260 & 0.0231 \\
1.21 & 0.0704 & 0.0577 & 0.0496 & 0.0281 & 0.0250 \\
1.27 & 0.0662 & 0.0573 & 0.0511 & 0.0296 & 0.0265 \\
1.30 & 0.0667 & 0.0579 & 0.0518 & 0.0302 & 0.0271 \\
1.31 & 0.0669 & 0.0581 & 0.0519 & 0.0304 & 0.0273 \\
1.50 & 0.0686 & 0.0602 & 0.0544 & 0.0331 & 0.0298 \\
\hline
\end{tabular}




\section{Chapter 3}

\section{Incomplete Models}

In chapter 3, we concentrate on the models when missing some information about our data. Some motivation about right censoring model, when using scaled Laplace transform is given in section 3.1. In subsection 3.1.1 we study the local properties of the estimated pdf. In subsection 3.1.2, we investigate the finite sample properties of the right censoring estimators. Recovering the cdf of current status model is demonstrated in Section 3.2. Moreover, in order to achieve this task two separate cases are investigated in this (a) the distribution of $X$ is known (b) the distribution of $X$ is unknown. The upper bounds for the uniform rate of convergence for the cdf is derived. Also, we investigate the asymptotic behavior of cdf. The estimation of the pdf of length baised model is introduced in Section3.3. 


\subsection{Right censored model}

Suppose $X_{1}, X_{2}, \ldots, X_{n}$ is a sequence of independent, non-negative random variables with common continuous distribution $F$. Let $C_{1}, C_{2}, \ldots, C_{n}$ be another sequence of independent, non-negative random variables with common right continuous distribution function $G$, which is known, and $\bar{G}=1-G$. Also, assume $\left\{X_{i}\right\}_{1}^{n}$ and $\left\{C_{i}\right\}_{1}^{n}$ are mutually independent. We will refer to $X_{i}$ as observed times and $C_{i}$ as censoring times. Under the random censorship model, we are only able to observe the smallest value among $X_{i}$ and $C_{i}$ and an indicator that $X_{i}$ is smaller or equal to $C_{i}$. Namely, the data represents the sequence of bivariate random variables $\left(Z_{i}, \delta_{i}\right), i=1, \ldots, n$ with $Z_{i}=\min \left(X_{i}, C_{i}\right)$, and $\delta_{i}=I\left\{X_{i} \leq C_{i}\right\}$ for $i=1,2, \ldots, n$. Such model is known as the rightcensored (R-C).

Let us denote by $H$ the cdf of non-censored $\left(Z_{i}, \delta_{i}\right)$ 's, that is,

$$
H(u)=P\left(Z_{i} \leq u, \delta_{i}=1\right)=\int P\left(X_{i} \wedge C_{i} \leq u, X_{i} \leq C_{i} \mid X_{i}=y\right) d F(y) .
$$

Since $X_{i}$ 's are independent from $C_{i}$ 's, we can write

$$
H(u)=\int P\left(y \wedge C_{i} \leq u, y \leq C_{i}\right) d F(y)=\int_{0}^{u} P\left(C_{i} \geq y\right) d F(y)=\int_{0}^{u} \bar{G}(y) d F(y) .
$$

By differentiating both sides of previous equation, we obtain

$$
d H(u)=\bar{G}(u) d F(u)
$$


i.e.,

$$
d F(u)=\frac{d H(u)}{\bar{G}(u)} .
$$

Consider the estimated Laplace transform of $F$ that is based on the estimated $\tilde{H}$ in the right hand side of (3.2). Namely, let:

$$
\tilde{\mu}_{j}(F)=\int_{\mathbf{R}_{+}} e^{-j c u} \frac{d \tilde{H}(u)}{\bar{G}(u)} .
$$

Now, for each $x \in \mathbf{R}_{+}$, the following estimator for $f$ is suggested:

$$
\tilde{f}_{\alpha, b}^{R C}(x)=\frac{\alpha}{\alpha+1}\left(\mathfrak{B}_{\alpha}^{-1} \tilde{\mu}(F)\right)(x) .
$$

Here $\mathfrak{B}_{\alpha}^{-1}$ is defined according to (2.3).

Remark 1. Note, that if $\hat{F}$ is the empirical distribution function based on the $X_{i}$ 's, i.e., $\hat{F}(x)=\frac{1}{n} \sum_{i=1}^{n} I\left(X_{i} \leq x\right)$. Since we do not observe all $X_{i}$ 's, the corresponding version of the empirical Laplace transform $\hat{\mathcal{L}}_{j}(F)$ is:

$$
\hat{\mathcal{L}}_{j}(F)=\int_{\mathbf{R}_{+}} e^{-j(\ln b) t} d \hat{F}(t)=\frac{1}{n} \sum_{i=1}^{n} e^{-j(\ln b) X_{i}},
$$

On the contrary, we can evaluate

$$
\hat{\mu}_{j}(F)=\int_{\mathbf{R}_{+}} e^{-j c t} \frac{d \hat{H}(t)}{\bar{G}(t)}=\frac{1}{n} \sum_{i=1}^{n} e^{-j c Z_{i}} \frac{I\left\{X_{i} \leq C_{i}\right\}}{\bar{G}\left(Z_{i}\right)},
$$

where $\hat{H}$ is the empirical distribution function of $\left(Z_{i}, \delta_{i}\right)$ 's when $\delta_{i}=1$, i.e. when $X_{i}$ 's observed. 
Actually, the suggested estimator $\hat{f}_{\alpha, b}^{R C}$ obtained by plugging-in the expression (3.6) instead of $\tilde{\mu}(F)$ into the right-hand side of (3.4) is:

$$
\hat{f}_{\alpha, b}^{R C}(x)=\frac{\alpha}{\alpha+1}\left(\mathfrak{B}_{\alpha}^{-1} \hat{\mu}(F)\right)(x), x \in \mathbb{R}_{+} .
$$

After some algebra we can write:

$$
\hat{f}_{\alpha, b}^{R C}=\frac{\left[\alpha b^{-x}\right] \ln b}{\alpha+1} \frac{1}{n} \sum_{i=1}^{n} \beta\left(b^{-Z_{i}},\left[\alpha b^{-x}\right]+1, \alpha-\left[\alpha b^{-x}\right]+1\right) \frac{I\left\{X_{i} \leq C_{i}\right\}}{\bar{G}\left(Z_{i}\right)} .
$$

\subsubsection{Mean sequared error}

In this subsection we are going to investigate the MSE of estimator $\hat{f}_{\alpha, b}^{R C}(x)$ denote by $\Lambda(x)=f(x) / \bar{G}(x)$. The following statement is true.

Theorem 4. Under the assumption (2.6), we have

$$
\begin{aligned}
\operatorname{MSE}\left\{\hat{f}_{\alpha, b}^{R C}(x)\right\} & \leq n^{-4 / 5}\left(\left(\frac{2 b^{x}\left|f^{\prime}(x)\right|}{\ln b}+\frac{b^{2 x}\left|f^{\prime}(x)\right|}{2 \ln ^{2} b}+\frac{b^{x}\left|f^{\prime \prime}(x)\right|}{2 \ln b}\right)^{2}+\frac{\ln b \Lambda(x)}{\sqrt{\pi\left(b^{x}-1\right)}}\right) \\
& +o\left(n^{-4 / 5}\right),
\end{aligned}
$$

provided that we choose $\alpha=\alpha(n) \sim n^{2 / 5}$, as $n \rightarrow \infty$.

At first, let us investigate the asymptotic behavior of the bias and variance terms of $\hat{f}_{\alpha, b}^{R C}$ defined in (3.7). The following statement is true. 
Lemma 5. For each $x \in(0, \infty)$, for the bias and variance terms of $\hat{f}_{\alpha, b}^{R C}(x)$ we have, respectively:

$\left|\operatorname{Bias}\left\{\hat{f}_{\alpha, b}^{R C}(x)\right\}\right| \leq \frac{1}{\alpha+1}\left\{\frac{2 b^{x}\left|f^{\prime}(x)\right|}{\ln b}+\frac{b^{2 x}\left|f^{\prime}(x)\right|}{2 \ln ^{2} b}+\frac{b^{x}\left|f^{\prime \prime}(x)\right|}{2 \ln b}\right\}+o\left(\frac{1}{\alpha}\right)$,

and

$$
\operatorname{var}\left\{\hat{f}_{\alpha, b}^{R C}(x)\right\}=\frac{\sqrt{\alpha}}{n} \frac{\Lambda(x) \ln b}{\sqrt{\pi\left(b^{x}-1\right)}}+o\left(\frac{\sqrt{\alpha}}{n}\right),
$$

as $\alpha \rightarrow \infty$.

\section{Proof of Lemma 4:}

Note that using the representation (3.7) we have for the expected value of $\hat{f}_{\alpha, b}^{R C}(x)$ :

$$
\begin{gathered}
E\left\{\hat{f}_{\alpha, b}^{R C}\right\}(x)=\frac{\left[\alpha b^{-x}\right] \ln b}{(\alpha+1)} E\left\{\beta_{\alpha, x}^{*}\left(b^{-Z_{i}}\right) \frac{I\left\{X_{i} \leq C_{i}\right\}}{\bar{G}\left(Z_{i}\right)}\right\} \\
=\frac{\ln b \Gamma(\alpha+1)}{\Gamma\left(\left[\alpha b^{-x}\right]\right) \Gamma\left(\alpha-\left[\alpha b^{-x}\right]+1\right)} \int_{0}^{\infty}\left(b^{-t}\right)^{\left[\alpha b^{-x}\right]}\left(1-b^{-t}\right)^{\alpha-\left[\alpha b^{-x}\right]} \frac{f(t) \bar{G}(t)}{\bar{G}(t)} d t .
\end{gathered}
$$

After changing the integration variable in (3.11) we obtain

$$
f_{\alpha, b}^{R C}(x):=E\left\{\hat{f}_{\alpha, b}^{R C}(x)\right\}=\int_{0}^{1} \beta_{\alpha, x}(\tau) q(\tau) d \tau,
$$

i.e., the mean and the bias of $\hat{f}_{\alpha, b}^{R C}(x)$ have the same forms as that of $\hat{f}_{\alpha, b}(x)$ (see (2.17)). Hence we obtain $\left|\operatorname{Bias}\left\{\hat{f}_{\alpha, b}^{R C}(x)\right\}\right| \leq \frac{1}{\alpha+1}\left\{\frac{b^{x}\left|f^{\prime}(x)\right|}{\ln b}+\frac{b^{2 x}\left|f^{\prime}(x)\right|}{2 \ln ^{2} b}+\frac{b^{x}\left|f^{\prime \prime}(x)\right|}{2 \ln b}\right\}+o\left(\frac{1}{\alpha}\right)$, 
as $\alpha \rightarrow \infty$.

Similarly, combining (3.7) and (3.12), and applying the same argument used in derivations of (2.22)-(2.25) we will have, respectively:

$$
\begin{aligned}
& \frac{1}{n}\left\{\frac{\left[\alpha b^{-x}\right] \ln b}{(\alpha+1)}\right\}^{2} E\left\{\beta_{\alpha, x}^{* 2}\left(b^{-Z_{1}}\right) \frac{I\left\{X_{1} \leq C_{1}\right\}}{\left\{\bar{G}\left(Z_{1}\right)\right\}^{2}}\right\} \\
& =\frac{1}{n}\left\{\frac{\left[\alpha b^{-x}\right] \ln b}{(\alpha+1)}\right\}^{2}\left(\frac{\Gamma(\alpha+2)}{\Gamma\left(\left[\alpha b^{-x}\right]+1\right)\left(\alpha-\left[\alpha b^{-x}\right]+1\right)}\right)^{2} \\
& \quad \times \int_{0}^{\infty}\left(b^{-y}\right)^{2\left[\alpha b^{-x}\right]}\left(1-b^{-y}\right)^{2 \alpha-2\left[\alpha b^{-x}\right]} \frac{f(y)}{\bar{G}(y)} d y \\
& =\xi_{\alpha}(x) \int_{0}^{1} \beta\left(\tau, 2\left[\alpha b^{-x}\right], 2 \alpha-2\left[\alpha b^{-x}\right]+1\right) \frac{q(\tau)}{\bar{G}(\phi(\tau))} d \tau,
\end{aligned}
$$

and

$$
\begin{aligned}
& \frac{1}{n}\left(\frac{\left[\alpha b^{-x}\right] \ln b}{\alpha+1}\right)^{2}\left(E \beta_{\alpha, x}^{*}\left(b^{-Z_{1}}\right) \frac{I\left\{X_{1} \leq C_{1}\right\}}{\bar{G}\left(Z_{1}\right)}\right)^{2} \\
& =\frac{1}{n}\left(\frac{\ln b \Gamma(\alpha+1)}{\Gamma\left(\left[\alpha b^{-x}\right]\right) \Gamma\left(\alpha-\left[\alpha b^{-x}\right]+1\right)}\right)^{2}\left(\int_{0}^{1} \tau^{\left[\alpha b^{-x}\right]-1}(1-\tau)^{\alpha-\left[\alpha b^{-x}\right]} q(\tau) \frac{d \tau}{\ln (b)}\right)^{2} \\
& =\frac{1}{n}\left(\int_{0}^{1} \beta_{\alpha, x}(\tau) q(\tau) d \tau\right)^{2}=\frac{1}{n}\left(f(x)+O\left(\frac{1}{\alpha}\right)\right)^{2}, \text { as } \alpha \rightarrow \infty .
\end{aligned}
$$

Again, since $\left\{\beta\left(\cdot, 2\left[\alpha b^{-x}\right], 2 \alpha-2\left[\alpha b^{-x}\right]+1\right), \alpha \in \mathbb{N}\right\}$ in (3.13) represents a $\delta$-sequence at $b^{-x}$, and the value of $q(\tau) / \bar{G}(\phi(\tau))$ at $\tau=b^{-x}$ 
is $\Lambda(x)=f(x) / \bar{G}(x)$, we arrive at

$$
\operatorname{var}\left\{\hat{f}_{\alpha, b}^{R C}(x)\right\}=\frac{\sqrt{\alpha}}{n} \frac{\Lambda(x) \ln b}{\sqrt{\pi\left(b^{x}-1\right)}}+o\left(\frac{\sqrt{\alpha}}{n}\right)
$$

as $\sqrt{\alpha} / n \rightarrow 0, \alpha, n \rightarrow \infty$.

Finally, we can get the upper bound for MSE of $\hat{f}_{\alpha, x}^{R C}(x)$ :

$$
\begin{aligned}
\operatorname{MSE}\left\{\hat{f}_{\alpha, b}^{R C}(x)\right\} & \leq \frac{1}{(\alpha+1)^{2}}\left(\frac{2 b^{x}\left|f^{\prime}(x)\right|}{\ln b}+\frac{b^{2 x}\left|f^{\prime}(x)\right|}{2 \ln ^{2} b}+\frac{b^{x}\left|f^{\prime \prime}(x)\right|}{2 \ln b}\right)^{2} \\
+ & \frac{\sqrt{\alpha}}{n} \frac{\Lambda(x) \ln b}{\sqrt{\pi\left(b^{x}-1\right)}}+o\left(\frac{\sqrt{\alpha}}{n}\right)
\end{aligned}
$$

as $\sqrt{\alpha} / n \rightarrow 0, \alpha, n \rightarrow \infty$. Choosing in (3.15) parameter $\alpha \sim n^{2 / 5}$, we derive (3.8).

\subsubsection{Simulation}

To evaluate the finite sample performance of the proposed probability density of right censored model, $f_{\alpha, b}^{R C}$, the survival time follows known distribution, and the censoring times generated from different known distribution. We consider two different sample sizes $n=200,500$ with different order of $\alpha$ and $b$. We analyze the average of $L_{2}$ - and supnorms, as a measure of errors of the estimator. They are defined as 
follows:

$$
\begin{aligned}
& d_{\alpha, b}=\frac{1}{R} \sum_{r=1}^{R}\left(\frac{1}{m} \sum_{j=1}^{m}\left(\hat{f}_{\alpha, b}^{R C}\left(x_{j}\right)-f\left(x_{j}\right)\right)^{2}\right)^{1 / 2}, \\
& d_{\alpha, b}^{*}=\frac{1}{R} \sum_{r=1}^{R} \max _{j \leq m}\left|\hat{f}_{\alpha, b}^{R C}\left(x_{j}\right)-f\left(x_{j}\right)\right|,
\end{aligned}
$$

respecively, where the number of replication $R=50$ and $\left\{x_{j}, j=1, \ldots, m\right\}$ represents the partition of support of $f$. Moreover, in this section we will compare the performance of density estimate $f_{\alpha, b}^{R C}$ with the kernel density estimation $\hat{f}_{h}$ constructed by means of the so-called KaplanMeier estimator $F^{K M}$ of $F$ (see [5], [13], [25], and [33]):

$$
\hat{f}_{h}(x)=\frac{1}{h} \int_{-\infty}^{\infty} K\left(\frac{x-y}{h}\right) d F^{K M}(y)
$$

Here the Kaplan-Meier estimator $F^{K M}$ is defined as follows:

$$
1-F^{K M}(u) \begin{cases}\Pi_{i: Z(i) \leq u}\left(\frac{n-i}{n-i+1}\right)^{\delta_{(i)}}, & , u<Z_{(n)} \\ 0 \quad, u \geq Z_{(n)}\end{cases}
$$

and $Z_{i}=\min \left(X_{i}, C_{i}\right), \delta_{i}=I\left\{X_{i} \leq C_{i}\right\}$ for $i=1,2, \ldots, n$. In the above formula $\delta_{(i)}$ is the mark corresponding to $Z_{(i)}$. Besides, we evaluate the performance of our construction based on the scaled Laplace transform inversion, and compare with gamma kernel density estimator studied in [7]. Corresponding Average $L_{2}$-error is defined in a similar way as $d_{\alpha, b}$ from (3.16):

$$
d_{h}=\frac{1}{R} \sum_{r=1}^{R}\left(\frac{1}{m} \sum_{j=1}^{m}\left(\hat{f}_{h}\left(x_{j}\right)-f\left(x_{j}\right)\right)^{2}\right)^{1 / 2} .
$$


Example 2. We have generated different samples of independent random samples of $X_{i}$ 's and $C_{i}$ 's, where $X \sim \operatorname{Lognormal}(1,1)$ and $C \sim$ $\operatorname{Gamma}(3,3)$. For example, we considered $n=200,500$, and estimated the density function $f$ by means of using the empirical Laplace transform $\hat{\mu}_{t}(F)$ defined in equation (3.6).

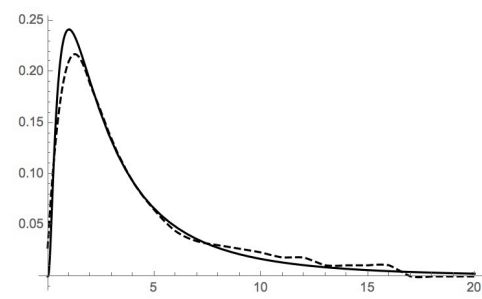

(a)

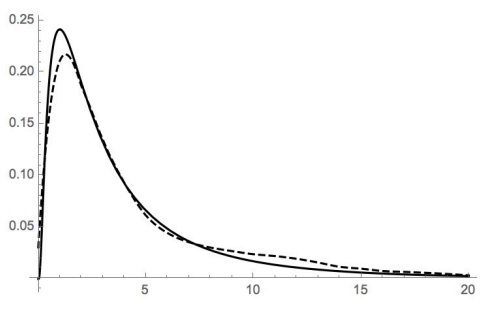

(b)

Figure 3.1: Estimated $\hat{f}_{\alpha, b}$ (dashed curve) with (a) $\alpha=40, b=1.30$, (b) with $\alpha=$ $80, b=1.13$, The true $f$ (solid curve).

In Table 3.1 we recorded the Average $L_{2}$-errors $d_{\alpha, b}\left(\times 10^{2}\right)$, when $X \sim \log$-normal $(1,1)$ and $C \sim \operatorname{Gamma}(3,3)$ for different choices of $\alpha, b$, and sample sizes $n=200,500$. From this table we can say that the optimal values of $b$ are: $b=1.30,1.13$, and 1.10 when $\alpha=40,80$, and 100 , respectively.

The Figure 3.1 displays the estimated density function $\hat{f}_{\alpha, b}^{R C}$ when $\alpha=40,80$ and $b=1.13,1.30$ and the sample size $n=500$.

Example 3. In this example in order to prove the the optimal result that was obtained from Table 3.1. We validate the optimal $\alpha$ and $b$ in several graphs. In Figure $3.2 X \sim \operatorname{Exponential(2/3)\text {and}}$ 
Table 3.1: Records of Average $L_{2}$-errors $d_{\alpha, b}\left(\times 10^{2}\right)$, when $X \sim \log$-normal $(1,1)$ and $C \sim \operatorname{Gamma}(3,3)$

\begin{tabular}{|c||c|c|c||c|c|c|}
\hline \multicolumn{1}{|c||}{} & \multicolumn{3}{c|}{$\mathrm{n}=200$} & \multicolumn{3}{c|}{$\mathrm{n}=500$} \\
\hline $\mathrm{b}$ & $\alpha=40$ & $\alpha=80$ & $\alpha=100$ & $\alpha=40$ & $\alpha=80$ & $\alpha=100$ \\
\hline 1.03 & 2.87 & 1.81 & 1.61 & 3.92 & 1.81 & 1.89 \\
1.10 & 1.64 & 1.52 & 1.45 & 1.29 & 1.03 & 1.09 \\
1.13 & 1.49 & 1.66 & 1.61 & 1.50 & 0.97 & 1.16 \\
1.23 & 1.53 & 1.85 & 1.95 & 1.23 & 1.17 & 1.23 \\
1.30 & 1.60 & 2.17 & 1.82 & 1.06 & 1.20 & 1.32 \\
1.80 & 1.71 & 2.14 & 2.22 & 1.27 & 1.34 & 1.27 \\
\hline
\end{tabular}

$C \sim$ Exponential(1/4). Using these parameters we will have about 25 percent of censored observations.

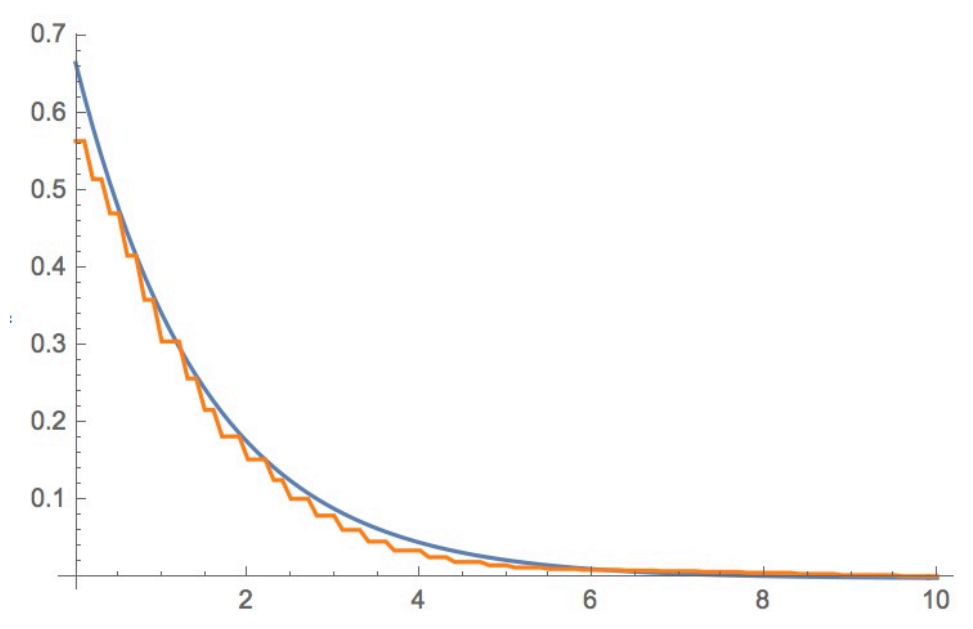

Figure 3.2: Estimated $\hat{f}_{\alpha, b}^{R C}$ (orange) and the true function $f$ (blue) with $(b=1.30, \alpha=$ 40), $n=500$.

In Figure $3.3 X \sim \operatorname{Lognormal}(1,2)$ and $C \sim \operatorname{Exponential}(2 / 3)$ with the same parameters as in previous Figure. Here we will have about 25 percent of censored observations. 


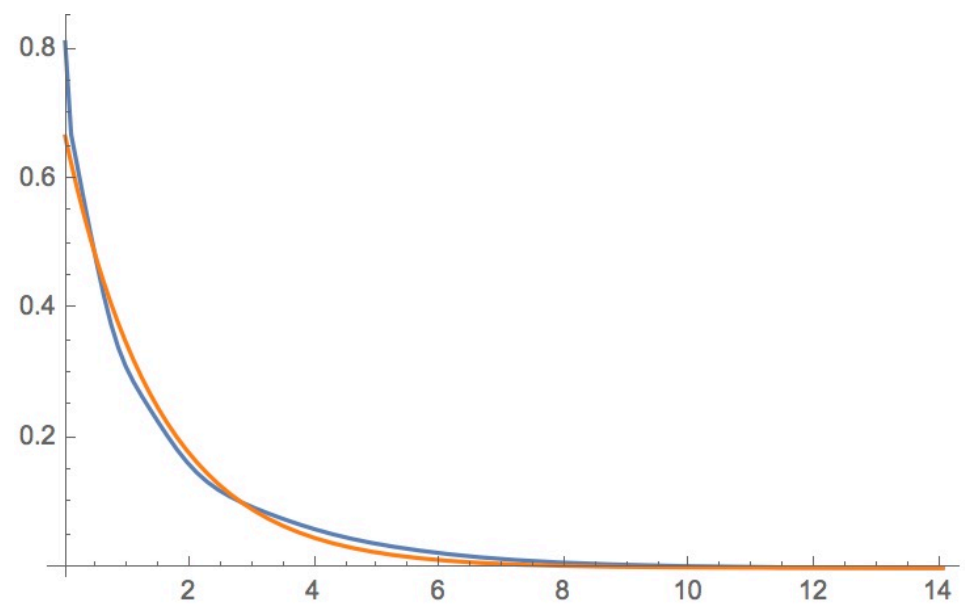

Figure 3.3: Linearly interpolated estimate $\hat{f}_{\alpha, b}^{R C}$ (orange), and the true function $f$ (blue) with $(b=1.30, \alpha=40), \mathrm{n}=500$.

Example 4. As a continuation of Example 2 we compared our $\hat{f}_{\alpha, b}^{R C}$ with kernel density using Kaplan-Meier estimator for right censored data defined in (3.16). Here we consider the Gaussian kernel. Figure 3.4 displays the true density $f, f_{h}$, and $\hat{f}_{\alpha, b}^{R C}$ based on the bandwidth $h \sim n^{-1 / 5}$ and the optimal $b=1.10$ and $\alpha=100$. 


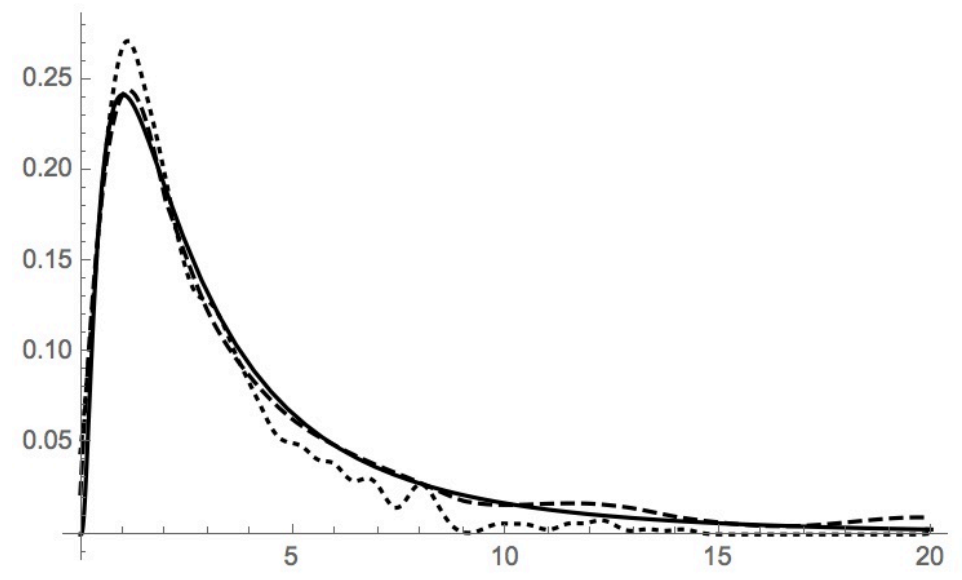

Figure 3.4: Estimates $\hat{f}_{\alpha, b}^{R C}$ (dashed), $\hat{f}_{h}$ (dotted), true curve $f$ (solid) with $(b=$ $1.10, \alpha=100)$.

Table 3.2 records the errors in sup-norm of estimator $\hat{f}_{\alpha, b}^{R C}$ evaluated in the case when $X \sim \operatorname{Lognormal}(1,1)$ and $C \sim \operatorname{Gamma}(3,3)$. Here, the optimal $b=1.13$ and three different values of $\alpha$ are considered.

Table 3.2: The Records of Average-errors in sup-norm for $\hat{f}_{h}$ and $\hat{f}_{\alpha, b}^{R C}$ when $b=1.13$

\begin{tabular}{|c|c|c|c|c|c|}
\hline \multicolumn{6}{|c|}{ Error } \\
\hline $\mathrm{n}$ & $\alpha=80$ & $\alpha=100$ & $\alpha=120$ & $d_{h}$ & $h$ \\
\hline 300 & 0.0252 & 0.0173 & 0.0159 & 0.0572 & 0.531 \\
500 & 0.02962 & 0.0201 & 0.01429 & 0.0489 & 0.501 \\
\hline
\end{tabular}

Example 5. In this example we compare our density with right censored data with Gamma kernel density proposed by [7] In Table 3.3 we took the optimal $b$ and $\alpha$ which are $(1.13,30)$ and sample sizes $n=125,250,500$ and compared with gamma density estimator $(\mathrm{G})$ by using models presented in [7], where the survival times 
and censoring times generated from Weibull distribution. Such that, $X \sim \operatorname{Weibull}(1.2,2)$ and for 10 percent $C \sim \operatorname{Weibull}(1.2,12.5), 25$ percent $C \sim \operatorname{Weibull}(1.2,4.9)$, and 50 percent $C \sim \operatorname{Weibull}(1.2,2)$.

Table 3.3: Records the Average $L_{2}$-errors $\left(\times 10^{2}\right)$ of $\hat{f}_{\alpha, b}^{R C}$ (with $\alpha=30, b=1.13$ ) and $G$-estimate proposed in [3]

\begin{tabular}{|c||c|c||c|c|c||c|}
\hline \multicolumn{1}{|c||}{} & \multicolumn{2}{c|}{$\mathrm{n}=125$} & \multicolumn{2}{c|}{$\mathrm{n}=250$} & \multicolumn{2}{c|}{$\mathrm{n}=500$} \\
\hline cens & $G$ & $\hat{f}_{\alpha, b}^{R C}$ & $G$ & $\hat{f}_{\alpha, b}^{R C}$ & $G$ & $\hat{f}_{\alpha, b}^{R C}$ \\
\hline 10 & 1.08 & 2.62 & 0.76 & 2.43 & 0.58 & 2.32 \\
25 & 1.65 & 2.75 & 1.56 & 2.51 & 1.5 & 2.34 \\
50 & 3.08 & 3.58 & 3.04 & 2.9 & 3.02 & 2.71 \\
\hline
\end{tabular}

In Table 3.3 we evaluated the Average $L_{2}$-norm as a measure of errors of the estimates. , The results were obtained using 1000 replications. By comparing the two estimators, our estimator was slightly better when the degree of censoring increased. For example, when $n=500$, degree of censoring 50 percent, our recored was 2.71 .

\section{Example 6.}

Let us generate $n=1000$ independent copies of $X \sim \operatorname{Gamma}(1,2)$ and $C \sim \operatorname{Gamma}(1,10)$. Consider the estimated survival function:

$$
\hat{S}_{\alpha, b}^{R C}(x)=\left(\mathcal{K}_{\alpha}^{-1} \hat{\mathcal{L}}(S)\right)(x),
$$

where $\mathcal{K}_{\alpha}^{-1}$ is defined in (1.3), and $\hat{\mathcal{L}}_{t}(S)=\frac{1}{n} \sum_{i=1}^{n} \frac{e^{-t c Z_{i}} I\left\{X_{i} \leq C_{i}\right\}}{S\left(Z_{i}\right)}$ is the estimated Laplace transform, $Z_{i}=\min \left(X_{i}, C_{i}\right)$. Denote by $\hat{S}^{K M}$ the 
Kaplan-Meier estimate of $S$ introduced in [25]. Figure 3.5 shows the curves of two estimators montioned above.

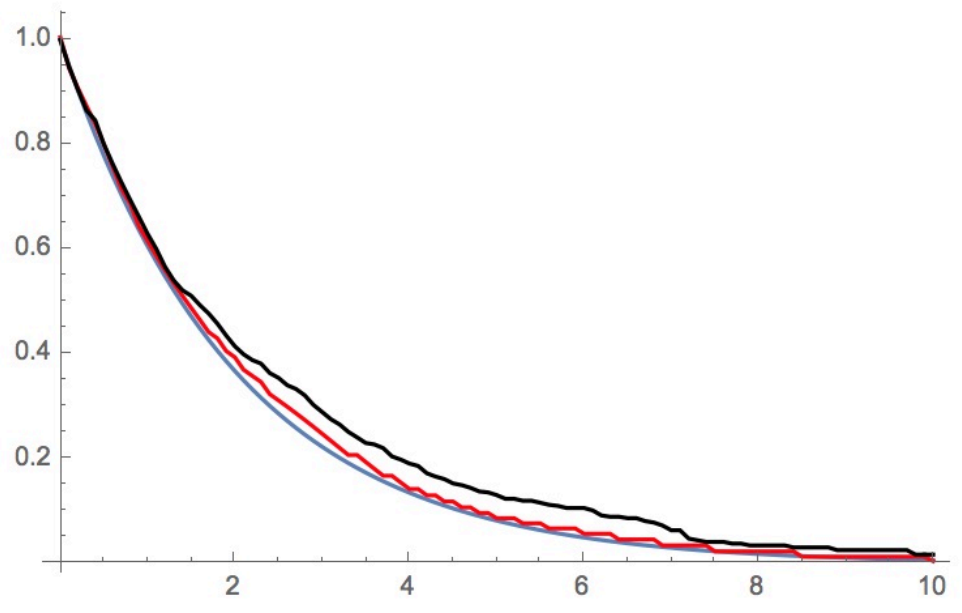

Figure 3.5: Estimation of $S$ (blue) by $\hat{S}_{\alpha, b}^{R C}$ (red) and $\hat{S}^{K M}$ (black) when $\alpha=80, b=$ 1.55 , and $n=1000$

\subsection{Current status model}

Let $\left(T_{1}, X_{1}\right),\left(T_{2}, X_{2}\right), \ldots,\left(T_{n}, X_{n}\right)$ be $\mathrm{n}$ iid pairs of random variables. For each $i, T_{i}$ distributed as $F_{T}, X_{i}$ is distributed as $\mathbb{P}_{X}$ and $T_{i}$ is independent of $X_{i}$. Also, $F_{T}, \mathbb{P}_{X}$ are continuous on the positive half line. We can think of $\mathrm{n}$ individuals with $T_{i}$ being the survival/ failure time and $X_{i}$ is the observation time for $\mathrm{i}$-th individual. For $\mathrm{i}$-th individual we observe $\left(X_{i}, I\left\{T_{i} \leq X_{i}\right\}\right)$. A function of our interest is the distribution of $F_{T}$. First, let us approximate function $h$ related to $F_{T}$ according to 
the following equation:

$$
h(x)=F_{T}(x) p(x)
$$

To approximate $h$ one can use the construction based on the moment of $h$ :

$$
\begin{aligned}
m_{1}(j) & =E\left(I\{T \leq X\} X^{j}\right) \quad j=0,1,2, \ldots, \alpha \\
& =\int x^{j} F_{T}(x) p(x) d x \\
& =\int x^{j} h(x) d x
\end{aligned}
$$

Namely, consider $h_{\alpha}=\mathfrak{B}_{\alpha}^{-1} m_{1}$ where $m_{1}=\left\{m_{1}(j), j=0, \ldots, \alpha\right\}$ and

$$
\left(\mathfrak{B}_{\alpha}^{-1} m\right)(x)=\frac{\Gamma(\alpha+1)}{\Gamma([\alpha x]+1)} \sum_{j=0}^{\alpha-[\alpha x]} \frac{(-1)^{j} m_{1}(j+[\alpha x])}{j !(\alpha-[\alpha x]-j) !}, \quad x \in[0,1]
$$

We suggest $F_{\alpha, T}=\frac{h_{\alpha}(x)}{p(x)}$

\subsubsection{Distribution of $X$ is known}

In this subsection we concentrate on the approximation of $F_{T}(x)$ when $X$ has known distribution. Also, we assume $F_{T}(x)$ has finite support, i.e, $\operatorname{supp}\left\{F_{T}\right\}=[0, L], \quad 0 \leq L<\infty$. For simplicity we consider $L=$ 1. In section 3.2.2 we explain the process when $X$ follows uniform distribution. To derive the upper bound for the approximation rate let 
us denote by $\Delta(h, \delta)=\sup _{|\tau-x| \leq \delta}|h(\tau)-h(x)|, 0<\delta<1$, the modulus of continuity of $h$.

Theorem 6. If $\inf _{0<x<1} p(x)=\gamma>0$, then $F_{\alpha, T} \longrightarrow_{u} F_{T}$ and $\left\|F_{\alpha, T}-F_{T}\right\| \leq \frac{\Delta(h, \delta)}{\gamma}+\frac{2}{\gamma(\alpha+2)} \frac{\|h\|}{\delta^{2}}$.

To prove Theorem 6 we need the following lemma.

Lemma 7. If $h$ is continuous on [0,1], then $h_{\alpha} \longrightarrow_{u} h$, and $\left\|h_{\alpha}-h\right\| \leq \Delta(h, \delta)+\frac{2\|h\|}{\delta^{2}(\alpha+2)}$

\section{Proof}

For approximated function $h_{\alpha}$ we can write:

$$
\begin{aligned}
& h_{\alpha}(x)=\frac{\Gamma(\alpha+2)}{\Gamma([\alpha x]+1)} \sum_{j=0}^{\alpha-[\alpha x]} \frac{(-1)^{j} E\left(I\{T<X\} X^{j}\right)}{j !(\alpha-[\alpha x]-j) !} \\
& =\frac{\Gamma(\alpha+2)}{\Gamma([\alpha x])} \sum_{j=0}^{\alpha-[\alpha x]} \frac{(-1)^{j}}{j !(\alpha-[\alpha x]-j) !} \int_{0}^{1} E\left(I\{T<X\} x^{j+[\alpha x]} \mid X=u\right) p(u) d u \\
& =\frac{\Gamma(\alpha+2)}{\Gamma([\alpha x]+1)} \sum_{j=0}^{\alpha-[\alpha x]} \frac{(-1)^{j}}{j !(\alpha-[\alpha x]-j) !} \int_{0}^{1} u^{j+[\alpha x]} E[I\{T \leq u\}] p(u) d u \\
& =\frac{\Gamma(\alpha+2)}{\Gamma([\alpha x]+1)} \sum_{j=0}^{\alpha-[\alpha x]} \frac{(-1)^{j}}{j !(\alpha-[\alpha x]-j) !} \int_{0}^{1} u^{j+[\alpha x]} F_{T}(u) p(u) d u \\
& =\frac{\Gamma(\alpha+2)}{\Gamma([\alpha x]+1)} \sum_{j=0}^{\alpha-[\alpha x]} \frac{(-1)^{j}}{j !(\alpha-[\alpha x]-j) !} \int_{0}^{1} u^{j+[\alpha x]} h(u) d u
\end{aligned}
$$

Changing the order of the summation and integration we get:

$$
h_{\alpha}(x)=\int_{0}^{1} \beta(u,[\alpha x]+1, \alpha-[\alpha x]+1) h(u) d u
$$


By splitting the integration of (3.22) into two parts we obtain:

$$
\begin{aligned}
& \left\|h_{\alpha}-h\right\|=\left|\int_{0}^{1}\{h(u)-h(x)\} \beta(u,[\alpha x]+1, \alpha-[\alpha x]+1) d u\right| \\
& \leq \sup _{0 \leq x \leq 1}\left(\int_{|u-x| \leq \delta}+\int_{|u-x|>\delta}\right)|h(u)-h(x)| \beta(u,[\alpha x]+1, \alpha-[\alpha x]+1) d u \\
& \leq \Delta(h, \delta)+R_{\alpha}
\end{aligned}
$$

Where $0<\delta<1$, and by using Chebyshev's inequality we get the following upper bound of $R_{\alpha}$.

$$
R_{\alpha} \leq 2\|h\| \sup _{0 \leq x \leq 1} \int_{0}^{1} \beta(u,[\alpha x]+1, \alpha-[\alpha x]+1) d u \leq \frac{2\|h\|}{\delta^{2}(\alpha+2)}
$$

By combining (3.23) and (3.24) the proof is complete. Now, we can proof theorem 6 .

$$
\frac{\left|h_{\alpha}(x)-h(x)\right|}{|p(x)|} \leq \frac{\Delta(h, \delta)}{\gamma}+\frac{2}{\gamma(\alpha+2)} \frac{\|h\|}{\delta^{2}}
$$

\subsubsection{Model 1: $X \sim p(x)=I_{(0,1)}(x)$}

Suppose $X \sim U(0,1)$, and consider (3.19) the approximation of $F_{T}$ is based on $F_{\alpha, T}=h_{\alpha}$

In the following Lemma we are going to prove the upper bound of $F_{T}$.

Lemma 8. If $X$ has uniform distribution then the upper bound of $F_{T}$ 
given by:

$$
\left\|h_{\alpha}-h\right\| \leq \Delta(h, \delta)+\frac{2\|h\|}{\delta^{2}(\alpha+2)}
$$

Example 7. Consider $X \sim U(0,1)$ and $T \sim \operatorname{Beta}(1,3)$.

The moment $m_{1}(j)=\frac{1}{j+1}+B(j+1,4)$. The form of the approximation $h_{\alpha}$ follows the equation (3.20).

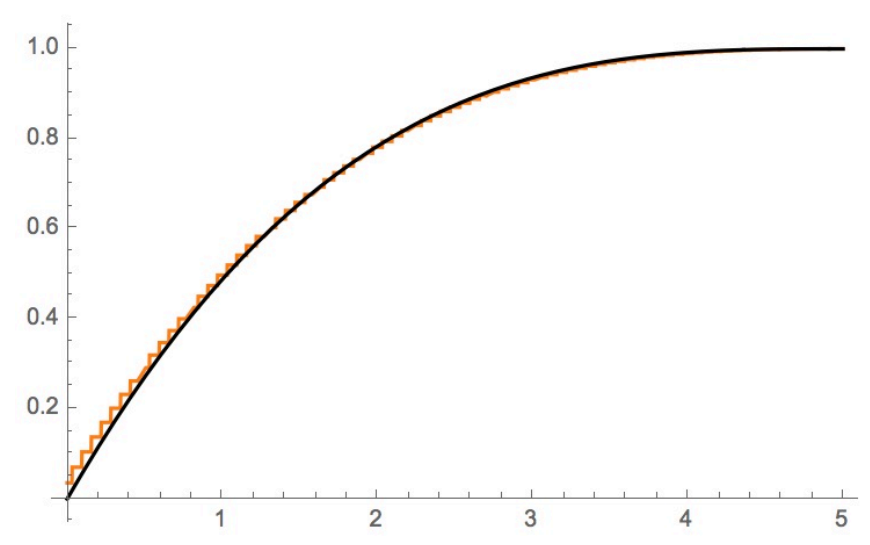

Figure 3.6: Approximed $F_{\alpha, T}$ (orange curve) with $\alpha=80$, the true $F_{T}$ (black curve).

In Table 3.4 the record of errors in sup-norm of $F_{\alpha, T}$ is presented. We can say as $\alpha$ increases the error decreases.

Table 3.4: Records of errors in sup-norm for $F_{\alpha, T}$

\begin{tabular}{|c|c|}
\hline$F_{\alpha, T}$ & sup-norm \\
\hline 20 & 0.0965055 \\
40 & 0.0600518 \\
60 & 0.0430906 \\
80 & 0.0335348 \\
100 & 0.0274312 \\
120 & 0.0232015 \\
140 & 0.0200994 \\
200 & 0.0143418 \\
\hline
\end{tabular}




\subsubsection{Model 2: $X \sim p(x), p(x)$ is known}

Let us assume that $X$ follows some known distribution with density function $p$. Assume $p$ has finite support, $\sup p\{p\}=[0,1]$. Given the sequence $m_{1}$, consider the approximation:

$$
F_{\alpha, T}(x)=\frac{1}{p(x)}\left(\mathfrak{B}_{\alpha}^{-1} m_{1}\right)(x),
$$

where $\mathfrak{B}_{\alpha}^{-1}$ is defined in (3.20), and the theorem 6 disscued the prperties about $F_{\alpha, T}$.

Example 8. Assume $F_{T}(x)=x^{2}$ and $p(x)=6 x(1-x)$. In this case:

$$
m_{1}(j)=\frac{6}{j+4}-\frac{6}{j+5}
$$

Figure 3.7 illustrate the approximation $F_{\alpha, T}$ and true curve $F_{T}$.

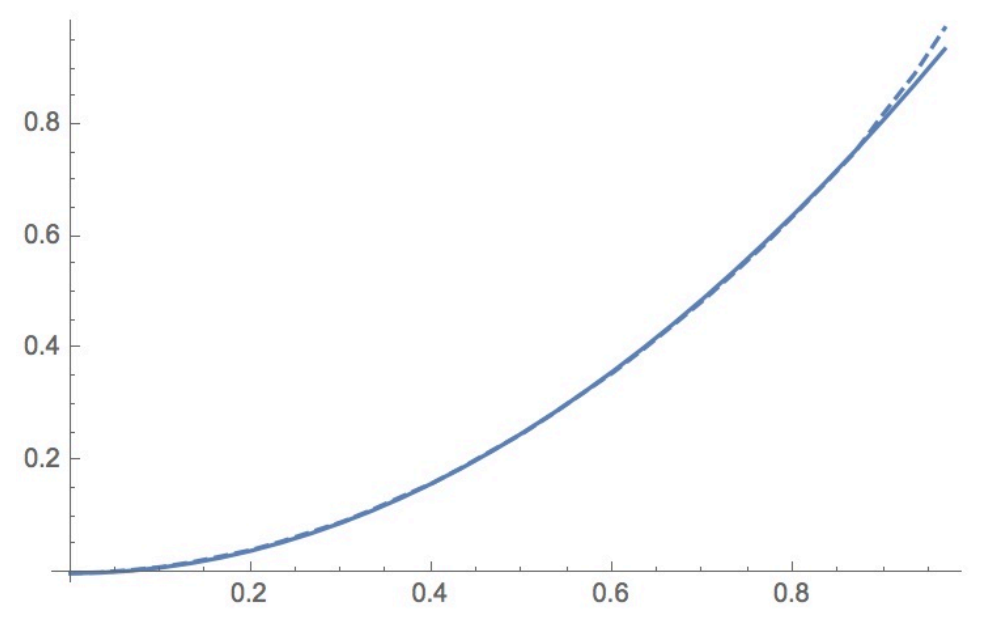

Figure 3.7: Approximed $F_{\alpha, T}$ (dashed curve ) with $\alpha=350$, the true $F_{T}$ (solid curve). 


\subsubsection{Model 3: $X \sim p(x)$, with $\operatorname{supp}\{p\}=\mathbb{R}_{+}$}

In this subsection we assume that the support of $p$ to be a positive half real line. Let us assume the following moment sequence $m_{0}=$ $\left\{m_{0}(j), j=0, \ldots, \alpha\right\}$ is given. Here

$$
m_{0}(j)=E\left(I\{T \leq X\} e^{-c x j}\right), \quad c=\ln b .
$$

To approximate unknown $F_{T}$ we suggest to use $F_{\alpha, T}$ defined as follows:

$$
F_{\alpha, T}(x)=\frac{1}{p(x)}\left(\mathfrak{B}_{\alpha}^{-1} m_{0}\right)(x) .
$$

Example 9. Assume $X \sim \operatorname{Exp}(\lambda)$ with $\lambda=2$, and the cdf of $T$ is also exponential with parameter $\lambda_{1}=2$. The momemnt sequance $m_{0}(j)$ given by:

$$
m_{0}(j)=\frac{\lambda}{\lambda+j}-\frac{\lambda}{\lambda+\lambda_{1}+j} .
$$

The Figure 3.8 represents the behavior of $F_{T}$ and $F_{\alpha, T}$ with parameters $\alpha=40$ and $b=2.748$. From the graph one can observe that the approximated curve $F_{\alpha, T}$ coinsides with the true $F_{T}$ curve. 


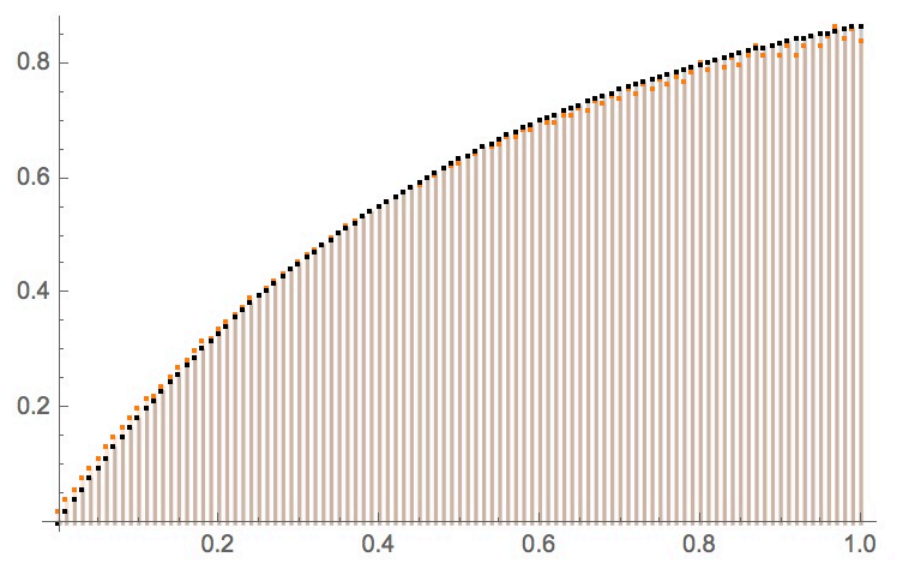

Figure 3.8: Approximed curve (orange) $F_{\alpha, T}$ with $\alpha=40, b=2.748$, the true $F_{T}$ (dashed black).

We construct the Table 3.5 by using the sup-norm error, and we find out the smallest error occure at 0.1307 , when $\alpha=40$ and $b=2.748$

Table 3.5: Records of sup - norm-errors $d_{\alpha, b}$

\begin{tabular}{|c||c|c|c|c|c|}
\hline \multicolumn{1}{|c||}{} & \multicolumn{5}{|c|}{ Error } \\
\hline$b, \alpha$ & 30 & 40 & 50 & 80 & 100 \\
\hline 1.53 & 15.1726 & 14.7917 & 14.5129 & 14.3020 & 14.2125 \\
2.5 & 0.7551 & 0.6651 & 0.5794 & 0.5108 & 0.6695 \\
2.7 & 0.9989 & 0.9994 & 0.9996 & 0.9998 & 0.9999 \\
2.756 & 0.2333 & 0.13402 & 0.1699 & 0.5414 & 0.6754 \\
2.748 & 0.2300 & 0.1307 & 0.1715 & 0.5417 & 0.6755 \\
2.8 & 0.9986 & 0.9992 & 0.9994 & 0.9997 & 0.9998 \\
3.0 & 0.9978 & 0.9987 & 0.9991 & 0.9996 & 0.9997 \\
\hline
\end{tabular}

\subsubsection{Distribution of $X$ is unknown}

In this section we assume that the distribution of $X$ is unknown, and $\operatorname{supp}\{p\}=[0, L], L \leq \infty$. To approximate $F_{T}$, assume for simplicity 
of notations that $L=1$, and consider two moment sequences $m_{k}=$ $\left\{m_{k}(j), j=0, \ldots, \alpha\right\}, k=1,2$, where

$$
m_{1}(j)=E\left(I\{T \leq X\} X^{j}\right)
$$

and

$$
m_{2}(j)=E\left(X^{j}\right)
$$

Given the sequence $m_{2}$, the unknown $p$ can be recovered by:

$$
p_{\alpha}(x)=\left(\mathfrak{B}_{\alpha}^{-1} m_{2}\right)(x)
$$

Therefore, the approximation $F_{\alpha, T}$ of $F_{T}$ will have the form:

$$
F_{\alpha, T}(x)=\frac{h_{\alpha}(x)}{p_{\alpha}(x)}, \quad x \in[0,1] .
$$

Example 10. Assume that $X \sim \operatorname{Beta}(3,2)$ and $F_{T}(t)=t^{3}, t>0$. In this case:

$$
m_{1}(j)=\frac{12}{j+6}-\frac{12}{j+7}
$$

and

$$
m_{2}(j)=\frac{12}{j+3}-\frac{12}{j+4}
$$

The Figure 3.9 displays the curve of approximation $F_{\alpha, T}$ and the true distribution $F_{T}$. 


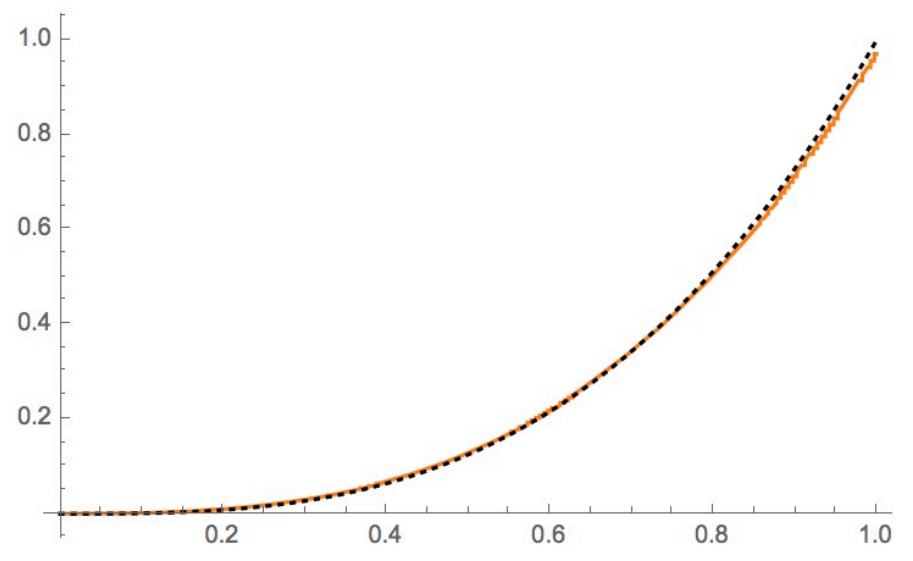

Figure 3.9: Approximion $F_{\alpha, T}$ (orange curve) with $\alpha=100$, the true $F_{T}$ (dotted curve).

\subsubsection{Estimation}

This subsection studies the local properties of the estimated $F_{\alpha, b}$ where the $\operatorname{supp}\{F\}$ is finite, twice differentiable, and $p(x)$ is known. We consider the empirical moment which is have the form:

$$
\hat{m}_{1}(j)=\frac{1}{n} \sum_{i=1}^{n} X_{i}^{j} I\left\{T_{i} \leq X_{i}\right\} \quad j=0,1, \ldots, \alpha .
$$

Therefore,

$$
\hat{F}_{\alpha, T}(x)=\frac{\hat{h}_{\alpha}(x)}{p(x)}=\frac{\left(\mathfrak{B}_{\alpha}^{-1} \hat{m}_{1}\right)(x)}{p(x)}
$$

where

$$
\hat{h}_{\alpha}(x)=\frac{\Gamma(\alpha+1)}{\Gamma([\alpha x]+1)} \sum_{j=0}^{\alpha-[\alpha x]} \frac{(-1)^{j} \hat{m}(j+[\alpha x])}{j !(\alpha-[\alpha x]-j) !}
$$


Also, remember that $\beta(u,[\alpha x]+1, \alpha-[\alpha x]+1)$, forms a $\delta$-sequance as $\alpha \rightarrow \infty$ with mean

$$
\eta_{\alpha}^{*}=\frac{[\alpha x]+1}{\alpha+2}
$$

and variance

$$
\sigma_{\alpha}^{*}=\frac{([\alpha x]+1)(\alpha-[\alpha x]+1)}{(\alpha+2)^{2}(\alpha+3)} \leq \frac{1}{\alpha+2}
$$

such that $\eta_{\alpha}^{*}-x=\frac{1-2 x}{\alpha+2}+\Delta_{1}(x)$ and $\sigma_{\alpha}^{*}-x=\frac{x(1-x)}{\alpha+1}+\Delta_{2}(x)$ where. $\Delta_{k}(x) \leq \frac{2}{(\alpha+2)^{2}}, k=1,2$ (Johnson et al. [22], Chen [10]).

Theorem 9. If $p(x)>0$ for $x \in(0,1)$, then the bias and MSE of $\hat{F}_{\alpha, T}(x)$ have representations:

$\operatorname{bias}\left\{\hat{F}_{\alpha, T}\right\}(x)=\frac{1}{p(x)(\alpha+2)}\left(h^{\prime}(x)(1-2 x)+\frac{1}{2} h^{\prime \prime}(x) x(1-x)\right)+o\left(\frac{1}{\alpha}\right), \alpha \rightarrow \infty$,

and

$$
\begin{gathered}
\operatorname{MSE}\left\{\widehat{F}_{\alpha, T}\right\}(x)=n^{-4 / 5}\left\{\left(\frac{1}{p(x)} h^{\prime}(x)(1-2 x)+\frac{1}{2} h^{\prime \prime}(x) x(1-x)\right)^{2}\right. \\
\left.+\frac{h(x)}{p^{2}(x) \sqrt{\pi x(1-x)}}\right\}+o\left(n^{-4 / 5}\right)
\end{gathered}
$$

if we choose $\alpha=\alpha(n) \sim n^{2 / 5}$, as $n \rightarrow \infty$.

\section{Proof:}

To derive the equations (3.35) we need to find $\frac{1}{p(x)}\left|E \hat{h}_{\alpha}(x)-h(x)\right|$.

$$
E \hat{h}_{\alpha}(x)=h_{\alpha}(x)=\int_{0}^{1} \beta(u,[\alpha x]+1, \alpha-[\alpha x]+1) h(u) d u
$$


Therefore, the bais of estimated $h_{\alpha}$ can be evaluated as :

$$
\begin{aligned}
\operatorname{bais}\left\{\hat{h}_{\alpha}(x)\right\} & =E\left\{\hat{h}_{\alpha}(x)\right\}-h(x)=h_{\alpha}(x)-h(x) \\
= & \int_{0}^{1} \beta(u,[\alpha x]+1, \alpha-[\alpha x]+1) h(u) d u-h(x) \\
= & \int_{0}^{1} \beta(u,[\alpha x]+1, \alpha-[\alpha x]+1)\{h(u)-h(x)\} d u
\end{aligned}
$$

Again using Taylor expansion for $h$ around $x$ and experssions of $\eta_{\alpha}^{*}$ and $\sigma_{\alpha}^{2 *}$ we obtain $(3.35)$.

$$
\text { Since } \widehat{F}_{\alpha, T}(x)=\frac{1}{n p(x)} \sum_{i=1}^{n} I\left\{T_{i} \leq X_{i}\right\} \beta\left(X_{i},[\alpha x]+1, \alpha-[\alpha x]+1\right),
$$

then

$$
\operatorname{var}\left\{\widehat{F}_{\alpha, T}\right\}=\frac{1}{n p^{2}(x)} \operatorname{var}\left\{I\left\{T_{1} \leq X_{1}\right\} \beta\left(X_{1},[\alpha x]+1, \alpha-[\alpha x]+1\right)\right\}
$$

But,

$$
\begin{aligned}
& \begin{aligned}
\frac{1}{n} E\left(I^{2}\{T \leq X\} \beta^{2}\left(X_{1},[\alpha x]+1, \alpha-[\alpha x]+1\right)\right)= \\
\quad=\frac{1}{n} \int_{0}^{1} \beta^{2}(u,[\alpha x]+1, \alpha-[\alpha x]+1) h(u) d u \\
\quad=\xi_{\alpha}^{*}(x) \int_{0}^{1} \beta(u, 2[\alpha x]+1,2 \alpha-2[\alpha x]+1) h(u) d u
\end{aligned} \\
& \text { Here, } \xi_{\alpha}^{*}(x)=\frac{1}{n}\left(\frac{\Gamma(\alpha+2)}{\Gamma([\alpha x]+1) \Gamma(\alpha-[\alpha x]+1)}\right)^{2} \frac{\Gamma(2[\alpha x]+1) \Gamma(2 \alpha-2[\alpha x]+1)}{\Gamma(2 \alpha+2)} \text {. Because } \\
& \int_{0}^{1} \beta(u, 2[\alpha x]+1,2 \alpha-2[\alpha x]+1) h(u) d u \rightarrow h(x) \text { as } \alpha \rightarrow \infty \text {. For each } \\
& x>0 \text { the order of magnitude } \xi_{\alpha}^{*}(x) \text { is }
\end{aligned}
$$

$$
\xi_{\alpha}^{*}(x)=\frac{\sqrt{\alpha}}{n \sqrt{\pi x(1-x)}}
$$


Thus,

$E\left(I^{2}\{T \leq X\} \beta^{2}\left(X_{1},[\alpha x]+1, \alpha-[\alpha x]+1\right)\right)=\frac{\sqrt{\alpha}}{n \sqrt{\pi x(1-x)}} h(x)+o\left(\frac{\sqrt{\alpha}}{n}\right)$,

as $\alpha \rightarrow \infty$. Now, consider

$$
\begin{aligned}
\frac{1}{n}(E(I\{T \leq X\} \beta(X,[\alpha x]+1, & \alpha-[\alpha x]+1)))^{2}= \\
& =\frac{1}{n}\left(\int_{0}^{1} \beta(u,[\alpha x]+1, \alpha-[\alpha x]+1) h(u) d u\right)^{2} \\
& =\frac{1}{n}\left(h(x)+O\left(\frac{1}{\alpha}\right)\right)^{2}, \text { as } \alpha \rightarrow \infty
\end{aligned}
$$

Finally, From (3.40) and (3.41) we obtain

$$
\operatorname{var}\left\{\widehat{F}_{\alpha, T}\right\}=\frac{\sqrt{\alpha}}{n \sqrt{\pi x(1-x)}} \frac{h(x)}{p^{2}(x)}+o\left(\frac{\sqrt{\alpha}}{n}\right),
$$

as $\sqrt{\alpha} / n \rightarrow 0, \alpha \rightarrow \infty$.

At this point we can write the MSE of $\widehat{F}_{\alpha, T}$ as:

$$
\begin{aligned}
\operatorname{MSE}\left\{\widehat{F}_{T}(x)\right\} & =\frac{1}{p^{2}(x)(\alpha+2)^{2}}\left(h^{\prime}(x)(1-2 x)+\frac{1}{2} h^{\prime \prime}(x) x(1-x)\right)^{2} \\
+ & \frac{\sqrt{\alpha}}{n \sqrt{\pi x(1-x)}} \frac{h(x)}{p^{2}(x)}+o\left(\frac{\sqrt{\alpha}}{n}\right)
\end{aligned}
$$

For the optimal rate, we may take

$$
\alpha=\alpha(n) \sim n^{2 / 5}
$$

By substituting $\alpha=n^{2 / 5}$ in (3.43), we find (3.36). 


\subsubsection{Simulation}

Example 11. In this example we have generated a random sample of $X$ from Uniform distribution, and $T$ from Beta distribution with parameters $p=3$ and $q=3$. We use the empirical moment defined in (3.32) and $\hat{f}_{\alpha, T}(x)=\frac{\hat{h}_{\alpha}(x)}{p(x)}=\frac{\left(\mathfrak{B}_{\alpha}^{-1} \hat{m}_{1}\right)(x)}{p(x)}$. The plot 3.10 shows the estimated $F_{\alpha, T}$.

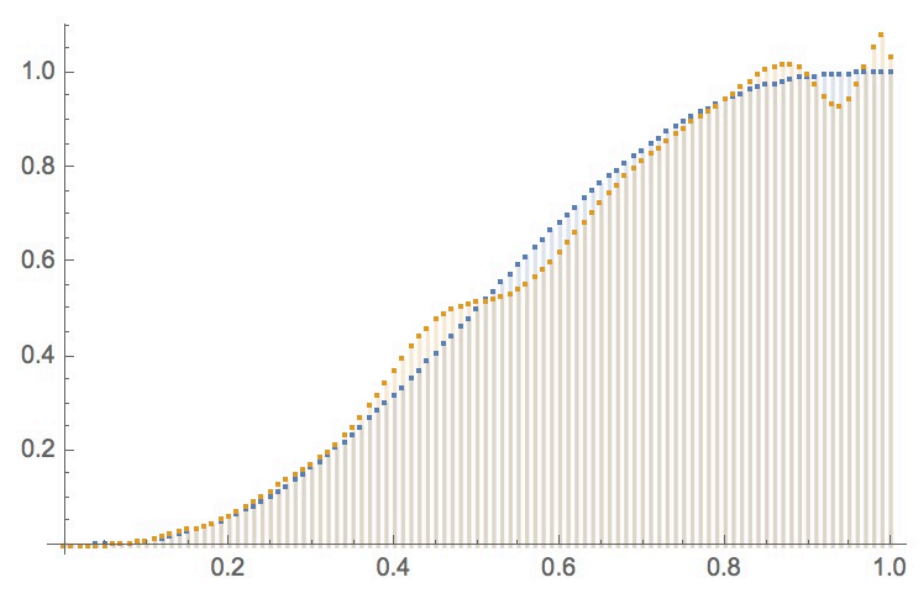

Figure 3.10: Estimation curve (orange) $F_{\alpha, T}$ with $\alpha=100$, the true $F_{T}$ (dotted blue).

Example 12. In this example we made a comparison between our estimator $F_{\alpha, T}$ with the estimator $\left(\Psi_{T}\right)$ introduced by [8]. As we have seen in Table 3.4 as $\alpha$ increases our model become more stable,so, we consider $\alpha=250$. Considering $X \sim \beta(4,6)$ and $T \sim \beta(4,8)$ and generating different sizes of random samples $n=60,200,500,1000$. 
Table 3.6: Comparison using MSE

\begin{tabular}{|c|c|c|}
\hline $\mathrm{n}$ & $M S E_{F_{\alpha, T}}$ & $M S E_{\Psi_{T}}$ \\
\hline 60 & 1.34 & 1.92 \\
200 & 0.39 & 0.83 \\
500 & 0.18 & 0.40 \\
1000 & 0.09 & 0.20 \\
\hline
\end{tabular}

In Table 3.6 the values of MSE are recorded. For our calculation we used the grid with points $u_{1}, u_{2}, \ldots, u_{J}$ from $[\mathrm{a}, \mathrm{b}], \mathrm{a}=0, \mathrm{~b}=0.5$ and $\mathrm{J}=500$. Namely, For MSE we use the following expression:

$$
M S E=\frac{b-a}{J} \sum_{j=1}^{J}\left[F\left(u_{j}\right)-\hat{F}_{T}\left(u_{j}\right)\right]^{2},
$$

where the number of replications $R=100$. The records form this Table 3.6 we can say that the proposed estimator performance better in terms of MSE.

\subsection{Length-biased model}

In this model the pdf $f$ and $\operatorname{cdf} F$ of a positive random variable $X$ is of actual interest, but one observe a random variable $Y$ with density

$$
g(y)=\frac{1}{W} w(y) f(y) ; \quad y \geq 0
$$

where the weight function $w$ and the total weight

$$
W=\int_{0}^{\infty} w(x) f(x) d x
$$


may not be known. In this chapter we assume we are given the empirical sequence of scaled Laplace transform by:

$$
\hat{\mathcal{L}}_{F}(t)=\int_{0}^{\infty} e^{-c t x} d \hat{F}_{n}(x)=\frac{W}{n} \sum_{i=1}^{n} \frac{e^{-c t Y_{i}}}{w\left(Y_{i}\right)}
$$

If we substitute (3.46) in (1.4). After some algebra we get:

$$
\hat{f}_{\alpha, b}^{L B}(x)=\frac{\left[\alpha b^{-x}\right] \ln (b) \Gamma(\alpha+2)}{(\alpha+1) \Gamma\left(\left[\alpha b^{-x}\right]+1\right)} \frac{W}{n} \sum_{i=1}^{n} \sum_{m=0}^{\alpha-\left[\alpha b^{-x}\right]} \frac{(-1)^{m} b^{-\left(m+\left[\alpha b^{-x}\right]\right) Y_{i}}}{m !\left(\alpha-\left[\alpha b^{-x}\right]-m\right) ! w\left(Y_{i}\right)}
$$

If $W$ is unknown one can estimated as follows:

$$
\hat{W}=\left(\frac{1}{n} \sum_{i=1}^{n} \frac{1}{w\left(Y_{i}\right)}\right)^{-1}
$$

and plug it into (3.47).

\subsubsection{Mean Squared Error of $f_{\alpha, b}^{L B}$}

In this section we investigate the bias, variance, and MSE of estimated $f_{\alpha, b}^{L B}$. For simplicity assume that $w(y)$ is the identity function.

Theorem 10. Under the assumption 2.6 the bais and MSE of $\hat{f}_{\alpha, b}^{L B}$ satisfies:

$\left|\operatorname{Bias}\left\{\hat{f}_{\alpha, b}^{L B}(x)\right\}\right| \leq \frac{1}{\alpha+1}\left\{\frac{2 b^{x}\left|f^{\prime}(x)\right|}{\ln b}+\frac{b^{2 x}\left|f^{\prime}(x)\right|}{2 \ln ^{2} b}+\frac{b^{x}\left|f^{\prime \prime}(x)\right|}{2 \ln b}\right\}+o\left(\frac{1}{\alpha}\right)$ as $\alpha \rightarrow \infty$. 


$$
\begin{aligned}
\operatorname{MSE}\left\{\hat{f}_{\alpha, b}^{L B}(x)\right\} & \leq n^{-4 / 5}\left(\left(\frac{2 b^{x}\left|f^{\prime}(x)\right|}{\ln b}+\frac{b^{2 x}\left|f^{\prime}(x)\right|}{2 \ln ^{2} b}+\frac{b^{x}\left|f^{\prime \prime}(x)\right|}{2 \ln b}\right)^{2}+\frac{W}{x} \frac{f(x) \ln b}{\sqrt{\pi\left(b^{x}-1\right)}}\right) \\
+ & o\left(\frac{1}{\alpha}\right)
\end{aligned}
$$

provided we choose $\alpha=\alpha(n) \sim n^{2 / 5}$, as $n \longrightarrow \infty$.

\section{Proof:}

$$
\begin{aligned}
E\left\{\hat{f}_{\alpha, b}^{L B}\right\}(x) & =\frac{\left[\alpha b^{-x}\right] \ln (b)}{\alpha+1} \frac{\Gamma(\alpha+2)}{\Gamma\left(\left[\alpha b^{-x}\right]+1\right)} \sum_{m=1}^{n} \frac{(-1)^{m} E\left(b^{-\left(m+\left[\alpha b^{-x}\right]\right) Y}\right)}{m !\left(\alpha-\left[\alpha b^{-x}\right]-m\right) !} \frac{W}{y} \\
& =W \frac{\left[\alpha b^{-x}\right] \ln (b)}{\alpha+1} \frac{\Gamma(\alpha+1)}{\left[\alpha b^{-x}\right]+1} \int_{0}^{\infty} \sum_{m=0}^{\alpha-\left[\alpha b^{-x}\right]} \frac{(-1)^{m} b^{-\left(m+\left[\alpha b^{-x}\right]\right) y}}{m !\left(\alpha-\left[\alpha b^{-x}\right]-m\right) !} \frac{g(y)}{y} d y
\end{aligned}
$$

After changing the order of summation and integration and some steps of algebra we obtain:

$E\left\{\hat{f}_{\alpha, b}^{L B}(x)\right\}=\frac{\left[\alpha b^{-x}\right] \ln (b)}{\alpha+1} \int_{0}^{\infty} \frac{W}{y} \beta\left(b^{-y},\left[\alpha b^{-x}\right]+1, \alpha-\left[\alpha b^{-x}\right]+1\right) g(y) d y$

Changing variables in (3.51) the expected of $\hat{f}_{\alpha, b}^{L B}$ will have the following form:

$$
E\left\{\hat{f}_{\alpha, b}^{L B}(x)\right\}=f_{\alpha, b}^{L B}(x)
$$

Note that the bias of $\hat{f}_{\alpha, b}^{L B}$ and $\hat{f}_{\alpha, b}$ defined in (2.20) of Chapter 2 coincide. For the variance of $\hat{f}_{\alpha, b}^{L B}$ using the similar steps applied in Chapter 
2 we get the following form:

$$
\operatorname{var}\left\{\hat{f}_{\alpha, b}^{L B}(x)\right\}=\frac{\sqrt{\alpha}}{n} \frac{W}{x} \frac{f(x) \ln b}{\sqrt{\pi\left(b^{x}-1\right)}}+o\left(\frac{\sqrt{\alpha}}{n}\right),
$$

as $\sqrt{\alpha} / n \rightarrow 0, \alpha, n \rightarrow \infty$.

Therefore, the MSE of $\hat{f}_{\alpha, b}^{L B}$ we have:

$$
\begin{aligned}
\operatorname{MSE}\left\{\hat{f}_{\alpha, b}^{L B}(x)\right\} & \leq \frac{1}{(\alpha+1)^{2}}\left\{\frac{2 b^{x}\left|f^{\prime}(x)\right|}{\ln b}+\frac{b^{2 x}\left|f^{\prime}(x)\right|}{2 \ln ^{2} b}+\frac{b^{x}\left|f^{\prime \prime}(x)\right|}{2 \ln b}\right\}^{2} \\
& +\frac{\sqrt{\alpha}}{n} \frac{W}{x} \frac{f(x) \ln b}{\sqrt{\pi\left(b^{x}-1\right)}}+o\left(\frac{\sqrt{\alpha}}{n}\right)
\end{aligned}
$$

Example 13. We simulated $n=1000$ copies of length-biased r.v.'s from gamma with $(4,1)$ parameters, $\alpha=25$ and $b=1.56$. The Figure 3.11 shows the estimated is closer to the true curve. 


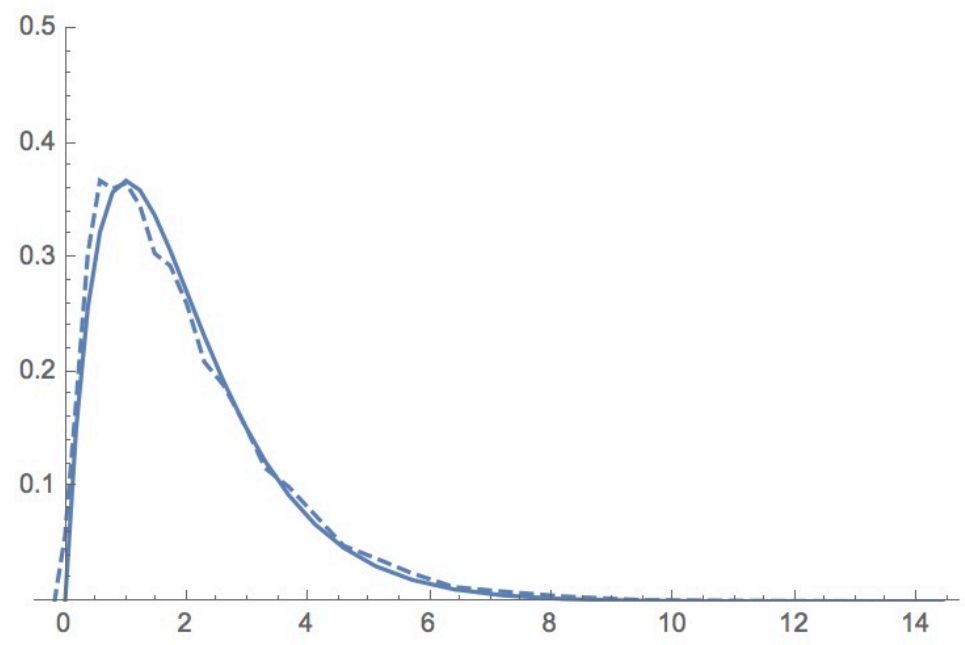

Figure 3.11: Estimated $f_{\alpha, b}^{L B}$ (dashed curve) and the true $f$ (solid curve), when $\alpha=$ $25, b=1.25$, and $n=1000$.

In Tabel 3.7 we generated $n=1000$ observations of $Y_{i}^{\prime} s$, where $y \sim$ gamma $(4,1)$. From this table we identify the optimal value of $\alpha$ and $b$ are 25 and 1.15 respectively.

Table 3.7: Records of errors in sup-norm

\begin{tabular}{|c||c|c|c|c|c|}
\hline \multicolumn{1}{|c||}{} & \multicolumn{5}{c|}{ Error } \\
\hline$b, \alpha$ & 25 & 30 & 32 & 34 & 36 \\
\hline 1.15 & 0.3582 & 0.3598 & 0.3621 & 0.3622 & 0.3607 \\
1.25 & 0.3259 & 0.3320 & 0.3348 & 0.3349 & 0.3385 \\
1.56 & 0.8864 & 0.9312 & 0.9408 & 0.9436 & 0.9468 \\
1.6 & 0.9526 & 1.003 & 1.012 & 1.0142 & 1.0205 \\
1.7 & 1.109 & 1.1715 & 1.1801 & 1.1811 & 1.1956 \\
1.9 & 1.4575 & 1.5174 & 1.5231 & 1.5196 & 1.5416 \\
\hline
\end{tabular}




\section{Chapter 4}

\section{Conclusion and FutureWork}

Several applications of the moment problem were considered in this dissertation. The methodology proposed in ([34],[38]) were applied to recover functions and distributions in defferent direct and indirect models. In this dissertation, several new moment-recovered estimations and approximations are proposed. In particular,the right censored, current status, length biased, and mean residual life time models are studied. Multiple examples were used to demonstrate the performance of the

proposed methods. In some models, the better results were achieved if compared with other traditional approches.

In Chapter 2, the estimation of the density function (the direct case) is investigated along with the asymptotic behavior of MSE in Section 2.1. Comparison of our estimates that are based on (1.4) with the traditional kernel density estimation is conducted. It is shown via the numerical and graphical evaluations the superiority of the former one. 
Also, the error of approximation of the mean residual life time function is studied.

In Chapter 3, the questions of approximation and estimation of the density and cumulative distribution functions in several incomplete models are studied. Namely, we considered: (a) right censorship (Section 3.1), (b) current status data (Section 3.2), and (c) length-biased models (Section 3.3).

In right-censored model we were able to derive the rate of convergence in terms of MSE for the density estimation. The simulation study was conducted to compare the proposed estimator with the one introduced in [7], as well as with smoothed kernel density construction based on Kaplan-Meier method.

In the model with Current Status Data, the approximation and estimation problem of underlying distribution function is investigated. The upper bound for approximation rate is derived. Two cases when the support of underlying function is bounded and unbounded from above are considered. Besides, two separate cases when the testing distribution is known and unknown are investigated. A simulation study was conducted to make the comparison with the method proposed in [8]. It is shown that our estimator performs better in terms of MSE.

The mean squared error of the estimated density function in the case of length biased model is derived. 
In the future work we will be concentrated on estimation of the mean residual life time function when the data is right-censored and length-biased. We will continue our work on nonparametric estimation in right-censored model when the distribution of censoring time is unknown. Also, we are planning to study the current status model in more complicated case when the observations are marked. 


\section{Bibliography}

[1] Ayer,M. Brunk,H. D. Ewing,G. M. Reid,W. T. and Silverman,E An empirical distribution function for sampling with incomplete information. Annals of Mathematical Statistics.(1955). 26: 641-647

[2] Ahmad, I. A. (1995). On multivariate kernel estimation for samples from weighted distributions. Statistics and Probability Letters, 22(2):pp. 121-129.

[3] Balkema, A.A and de Hann, L. Residual life time at great age, Annals of Probability.(1974) 2 (5), 792-804.

[4] Belkacem, A., Alexandre,B. Mean residual life estimation.Journal of Statistical Planning and Inference 132 (2005) 319.

[5] Blum, J. R. and Susarla, V. Maximal deviation theory of density and failure rate function estimates based on censored data. In 
Multivariate Analysis 5 (P. R. Krishnaiah, ed.) 213-222. NorthHolland,New York.

[6] Bouezmarni, T. and Rolin,J. Consistency of beta kernel density function estimator. The Canadian Journal of Statistics. (2003) vol. 31 no. 1 89-98.

[7] Bouezmarni, T. El Ghouch, and Mesfioui, M. Gamma kernel estimators for density and hazard rate of right censored data. Journal of Probability and Statistics. (2011).

[8] Brunel,E. Comte, F. Cumulative distribution function estimation under interval censoring case I. AMS Classification. (2008) $62 \mathrm{G} 05 ; 62 \mathrm{G} 20$.

[9] Borrajo,M.I ,Manteiga1, G.W.,Miranda,M. Bandwidth selection for kernel density estimation with length-biased data.(2016).

[10] Chen, S. X. (1999). Beta Kernel Estimators for Density Functions. Comput. Statist. Data Anal., 31, 131-145.

[11] Chen, S. X. (2000). Probability density function estimation using gamma kernels. Annals of the Institute of Statistical Mathematics. vol. 52, no. 3, 471-480. 
[12] Chaubey, Y.P. and Sen,P.K. On smooth estimation of mean residual life, Journal of Statistical Planning and Inference.(1999) 75, 223-236.

[13] Földes, A., Rejtö, L. and Winter, B.B. Strong consistency properties of nonparametric estimators for randomly censored data, II: Estimation of density and failure rate. Period. Math. Hungar 12. (1981) 15-29.

[14] Groeneboom, P. Asymptotics for interval censored observations. Technical Report 87-18, (1987). Department of Mathematics, University of Amsterdam.

[15] Groeneboom, P. and Wellner, J.A. Information bounds and nonparametric maximum likelihood estimation. Boston, Birkhäauser Verla $g(1992)$.

[16] Guess, F., Proschan, F. Mean residual life.(1988) In: Rao Krishnaiah, P.R., Rao, C.R. (Eds.), Handbook of Statistics, vol. 7. North-Holland, Amsterdam, pp. 215-224.

[17] Gill,R.D., Vardi,Y. Wellner, J.A. Large sample theory of empirical distribution in baised sampling models. The annals of statistics. (1988) Vol.16, No.13 1069-1112. 
[18] Jones, M.C. Simple boundary correction for kernel density estimation, Statistics and computing. (1993) vol.3, no. 3, 135-146.

[19] Jones, M.C. and Foster, P.J. (1996). Asimple nonnegative boundary correction method for kernel density estimation. Statistica Sinica. vol. 6, no. 4, 1005-1013.

[20] Jones, M. C. (1991). Kernel density estimation for length biased data. Biometrika, 78(3):pp. 511-519.

[21] Jones, M. C. and Karunamuni, R. J. (1997). Fourier series estimation for length biased data. Australian Journal of Statistics, 39(1):pp. 57-68.

[22] Johnson, N. L., Kotz, S. and Balakrishnan, N. (1994). Continuous Univariate Distributions. Wiley, New York.

[23] Hall,W.J.,Wellner, J.A. Mean residual life. In: Csörgó, M., Dawson, D.A., Rao, J.N.K., Saleh, A.K.Md.E. (Eds.), Statistics and Related Topics. (1981) North-Holland, Amsterdam, pp. 169-184.

[24] Huang, J. and Wellner, J. A. Asymptotic Normality of the NPMLE of Linear Functionals for Interval Censored Data, Case 1.(1995a).Statistica Neerlandica 49, 153 - 163. 
[25] Kaplan, E. L. and Meier, P. Nonparametric estimation for incomplete observations. J. Amer. Statist. Assoc. (1958) vol. 53 457481.

[26] Kotz, S., Shanbhag, D.N. Some new approaches to probability distributions. Adv. in Appl. Probab.(1980) 12, 903-921.

[27] Kleinbaum, D. and Klein, M. Survival Analysis,A self-Leraning Text.(2012).

[28] Kleiber, C. and Stoyanov, J. (2012). Multivariate distributions and the moment problem. Jour. of Multivariate Analysis. 113, 7-18.

[29] Lejeune, M. and Sarda, P. Smooth estimators of distribution and density functions. Computational Statistics and Data Analysis. (1992) vol. 14, no. 4, 457-471.

[30] Lin, G.D. (1997). On the moment problem. Statist. Probab. Lett, $35,85-90$.

[31] Marrom, J.S. and Rupprt, D. Transformation to reduce the boundary bais in kernel estimation. Journal of the Royal Statistical Society. (1994) vol. 56, no. 4, 653-671.

[32] Meilijson,I. Limiting properties of the mean residual lifetime function, Annals of Mathematical Statistics. (1972). 43 (1), 354357. 
[33] Mielniczuk, J. Some asymptotic properties of kernel estimators of a density function in case of censored data. Annual Statistic. (1986) 766-773.

[34] Mnatsakanov, R.M. (2008a). Hausdorff moment problem: Reconstruction of distributions. Statist. Probab. Letters, 78, 1612-1618.

[35] Mnatsakanov, R.M. (2008b). Hausdorff moment problem: Reconstruction of probability density functions. Statist. Probab. Letters, $78,1868-1877$.

[36] Mnatsakanov, R. M. (2011). Moment-recovered approximations of multivariate distributions: The Laplace transform inversion. Statist. Probab. Letters, 81, 1-7.

[37] Mnatsakanov,R. and Ruymgaart, F. H.(2005). Some result for moment-empirical cumulative distribution functions. 733-744.

[38] Mnatsakanov, R. and Sarkisian, K. (2013). A note on recovering the distributions from exponential moment. Applied Mathematics and Computation. 8730-8737.

[39] Müller, H. G. Smooth optimum kernel estimators near endpoints. Biometrika. (1991) vol. 78, no. 3, 521-530.

[40] Ruiz, J.M., Guillamòn, A. Nonparametric recursive estimator of residual life and vitality functions under mixing dependence 
conditions. Commun. Statist.- Theory and Methods.(1996) 4, 1999-2011.

[41] Silverman, B. (1986). Density Estimation for Statistics and Data Analysis. Chapman and Hall, New York.

[42] Shohat, J.A. and Tamarkin, J.D. (1943). The Problem of Moments. American Mathematical Society, Providence, RI.

[43] Stoyanov. J and Krein.J. Condition in Probabilistic moment problem. Bernoulli 6. (2000) 939-949.

[44] van de Geer, S. Hellinger-consistency of certain nonparametric likelihood estimators. Ann. Statist.(1993) 21, 14-44.

[45] Van Eeden, C. Maximum likelihood estimation of ordered probabilities.Proc. Kon. Ned. Akad. Wet. (1956). A59, 444 - 455.

[46] Van Eeden, C. Maximum likelihood estimation of partially ordered or completely ordered probabilities, I. Proc. Kon. Ned. Akad. Wet.(1957). A60, 128 - 136.

[47] Vardi, Y.(1982). Nonparametric estimation in the presence of length bias. The Annals of Statistics, 10(2):pp. 616-620.

[48] Yang, S. Functional estimation under interval censoring case 1. J. Statist. Plann. (2000). 89, 135-144. 
[49] Zhang, B. Some asymptotic result for kernel density estimation under random censorship. Bernoulli 2. (1996) 183-198. 